\title{
Ellerman bombs and UV bursts: reconnection at different atmospheric layers ${ }^{\star}$
}

\author{
Ada Ortiz ${ }^{1,2}$, Viggo H. Hansteen ${ }^{1,2}$, Daniel Nóbrega-Siverio ${ }^{1,2}$, and Luc Rouppe van der Voort $^{1,2}$ \\ 1 Rosseland Centre for Solar Physics, University of Oslo, PO Box 1029 Blindern, 0315 Oslo, Norway \\ e-mail: ada@astro.uio.no \\ 2 Institute of Theoretical Astrophysics, University of Oslo, PO Box 1029 Blindern, 0315 Oslo, Norway
}

Received 26 August 2019 / Accepted 20 October 2019

\begin{abstract}
The emergence of magnetic flux through the photosphere and into the outer solar atmosphere produces, amongst other dynamical phenomena, Ellerman bombs (EBs), which are observed in the wings of $\mathrm{H} \alpha$ and are due to magnetic reconnection in the photosphere below the chromospheric canopy. Signs of magnetic reconnection are also observed in other spectral lines, typical of the chromosphere or the transition region. An example are the ultraviolet (UV) bursts observed in the transition region lines of Si IV and the upper chromospheric lines of Mg II. In this work we analyze high-cadence, high-resolution coordinated observations between the Swedish $1 \mathrm{~m}$ Solar Telescope (SST) and the Interface Region Imaging Spectrograph (IRIS) spacecraft. H $\alpha$ images from the SST provide us with the positions, timings, and trajectories of EBs in an emerging flux region. Simultaneous, co-aligned IRIS slit-jaw images at 133 (C II, transition region), 140 ( $\mathrm{Si}$ IV, transition region), and 279.6 ( $\mathrm{Mg}$ II k, core, upper chromosphere) nm as well as spectroscopy in the far- and near-ultraviolet from the fast spectrograph raster allow us to study the possible chromospheric and transition region counterparts of those EBs. Our main goal is to study the possible temporal and spatial relationship between several reconnection events at different layers in the atmosphere (namely EBs and UV bursts), the timing history between them, and the connection of these dynamical phenomena to the ejection of surges in the chromosphere. We also investigate the properties of an extended UV burst and their variations across the burst domain. Our results suggest a scenario where simultaneous and co-spatial EBs and UV bursts are part of the same reconnection system occurring sequentially along a vertical or nearly vertical current sheet. Heating and bidirectional jets trace the location where reconnection takes place. These results support and expand those obtained from recent numerical simulations of magnetic flux emergence.
\end{abstract}

Key words. Sun: atmosphere - Sun: chromosphere - Sun: transition region - Sun: UV radiation

\section{Introduction}

The emergence of new magnetic flux from the solar interior into the photosphere and the regions above is a fundamental process as it facilitates the uplifting and renewal of magnetic flux in the solar atmosphere, and is a key player in the life cycle of the global magnetic field. A plethora of transient dynamical phenomena including surges, flares, jets, ultraviolet (UV) bursts, Ellerman bombs (EBs), and coronal mass ejections (CMEs) to mention but a few - occur when the newly emerged magnetic field interacts with the pre-existing (so-called ambient) field and/or with itself (see, e.g., Yokoyama \& Shibata 1995; Fletcher et al. 2011; Shibata \& Magara 2011; Moreno-Insertis \& Galsgaard 2013; Raouafi et al. 2016). The mentioned transient phenomena help the new field to rise and to push the old field aside by filling the chromosphere and corona, because they alleviate the mass that the rising field lines carry within them. With every reconnection event, mass is ejected (or drains downward) and that allows the magnetic field to attain coronal heights and form the long loops that make up the active region corona (see, e.g., Pariat et al. 2004). It also constitutes a significant input of mass and energy, which are impulsively released to the upper solar atmosphere and even to the solar wind.

The interaction between flux systems depends on the strength of the field involved, on the relative angle between

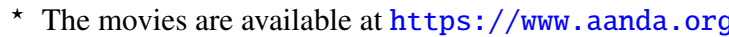

them, and on the height at which reconnection occurs (see, e.g., Shibata 1997; Archontis et al. 2004; Galsgaard et al. 2007; Isobe et al. 2007; MacTaggart et al. 2015). For instance, EBs are produced when reconnection happens at low altitudes while X-ray jets occur when the interaction takes place at greater heights.

Some excellent reviews covering the topic of flux emergence in depth, both from an observational and from a numerical point of view, are those by Archontis (2012), Cheung \& Isobe (2014), and Schmieder et al. (2014). In this work, we focus on two of the aforementioned transient dynamical phenomena related to flux emergence and reconnection, as well as on the possible relationship between them: EBs and UV bursts.

Ellerman bombs (Ellerman 1917) are defined by Vissers et al. (2015) as substantial brightenings of the extended wings of $\mathrm{H} \alpha$ in the absence of core brightening, which, at sufficient angular and temporal resolution, show rapid-flame morphology when viewed from the side. These authors extensively analyzed the properties of EBs, their relationship to other phenomena, and their visibility at other wavelengths in a series of papers (Watanabe et al. 2011; Vissers et al. 2013, 2015). In that series, these latter authors established that EBs are purely photospheric phenomena that trace the magnetic reconnection of strong opposite-polarity field concentrations in the low photosphere. Vissers et al. (2015) made it clear that many of the EBs found in the literature are actually not real EBs, but "pseudo-EBs" that also appear bright in the wings of $\mathrm{H} \alpha$ due to radiation escaping from deeper layers rather than heating by 
photospheric reconnection. According to these authors, care should be taken to ensure that features that appear bright in an $\mathrm{H} \alpha$ wing are indeed EBs and not simply facular brightenings, a warning already given by Ellerman (1917) himself.

Ultraviolet bursts (Peter et al. 2014) are defined, according to the recent review by Young et al. (2018), as compact ( $\leq 2^{\prime \prime}$, typically $1^{\prime \prime}$ ), transient (lifetimes between tens of seconds to over an hour), intense (but not related to flares) events observed in image sequences sampling UV bands. In addition, their Si IV line profiles present a complex shape with extended wings, large broadening and sometimes multiple peaks. UV bursts appear in locations where small magnetic features of opposite polarity collide, and do not generally show a co-spatial brightening in the coronal channels of the Solar Dynamics Observatory (SDO) Atmospheric Imaging Assembly (AIA) instrument. Different works consider different flavors of UV bursts, which may lead to different results. While Peter et al. (2014) analyze simple compact brightenings of about $1-2^{\prime \prime}$ in diameter, the multiwavelength study of Guglielmino et al. $(2018,2019)$ considers very extended brightenings of around $5^{\prime \prime}$ in diameter that are longer-lived (three hours) than previously reported bursts and present coronal counterparts in all passbands of SDO/AIA. These latter authors conclude that the reconnection site occurs higher in the atmosphere than usually found in UV bursts, which could explain the coronal counterparts. The magnetic topology above UV bursts will determine its visibility at coronal levels.

In recent times, there has been a debate in the literature about the relationship between these two particular phenomena - if any - and where in the atmosphere UV bursts form in relation to EBs. Many of the existing works that study this relationship advocate that UV bursts are hot pockets of gas originating very low in the solar atmosphere (even at upper photospheric heights) that would then be heated to transition region temperatures around $8 \times 10^{4} \mathrm{~K}$ (e.g., Peter et al. 2014; Vissers et al. 2015; Grubecka et al. 2016; Tian et al. 2016, 2018). Using He observations, and being the first to report on EB signatures in the $\mathrm{He} \mathrm{I}_{3}$ and He I $1083 \mathrm{~nm}$ lines, Libbrecht et al. (2017) derive EB temperatures of order $T \sim 2 \times 10^{4}-10^{5} \mathrm{~K}$ because they exhibit emission signals in neutral helium triplet lines. If the above studies are correct, current models of EBs need to be revised, as they are far from predicting such high photospheric temperatures. Indeed, models show that EBs cannot be reproduced if the photospheric temperature is too high (e.g., Reid et al. 2017; Hong et al. 2017). Recently, Vissers et al. (2019) found, from chromospheric spectral inversions, temperature enhancements of only a few thousand Kelvin around the height of the temperature minimum in co-spatial EBs and UV bursts. However, no satisfactory results could be achieved for coupled inversions that included both the Si IV and the chromospheric Ca II and Mg II lines at the same time. The inversion derived that the high temperatures in the lower atmosphere required to produce sufficient Si IV emission resulted in unrealistic synthetic Ca II and $\mathrm{Mg}$ II profiles, suggesting that EBs do not achieve transition region temperatures.

Peter et al. (2014) could not conclude on whether or not EBs and UV bursts were the same phenomena. Tian et al. (2016) claim that some of their UV bursts are also EBs and therefore form in the photosphere, which would be locally heated to $\sim 8 \times 10^{4} \mathrm{~K}$. The argument is given based on O IV emission, Mn I absorption in the wings of $\mathrm{Mg}$ II $\mathrm{k}$, deep absorption Ni II lines superimposed in the Si IV $139.4 \mathrm{~nm}$ profiles, compact brightenings in the AIA $170 \mathrm{~nm}$ passband, and enhanced emission in the Mg II wings, but not the core. These latter authors note that some other UV bursts in their data sets may originate in the chromosphere and have no connection to EBs.
Concerning the theoretical approach, Judge (2015), through $1 \mathrm{D}$ average atmospheric models, explained that the spectra of UV bursts have to have their origin, at least $550 \mathrm{~km}$ above the continuum photosphere. Recent 2.5D and 3D numerical simulations have found evidence that UV bursts are indeed formed in the chromosphere or above, and can possibly be the chromospheric counterparts of EBs.

In particular, by means of forward modeling of $2.5 \mathrm{D}$ magnetic flux emergence experiments, Nóbrega-Siverio et al. (2017) showed that UV burst-like Si IV profiles are obtained in the current sheet between the emerged plasma and the preexisting ambient field at coronal heights of 5-6 Mm above the photosphere. This model was also used by Rouppe van der Voort et al. (2017) to synthesize $\mathrm{Ca}$ II $\mathrm{K}$ and provide theoretical support to the profiles obtained with CHROMIS observations at UV bursts.

With respect to 3D models, Hansteen et al. (2017) analyze a flux emergence scenario in which reconnection between colliding cold magnetized bubbles is triggered at different heights, such that EBs are purely photospheric and reach temperatures of $T \sim 8-9 \times 10^{3} \mathrm{~K}$, UV bursts are a mid chromospheric phenomena (originating at $1.3 \mathrm{Mm}$ in the case shown in the paper) with temperatures of $T \sim 7-8 \times 10^{4} \mathrm{~K}$, and chromospheric microflares (at $1.8 \mathrm{Mm}$ ) reach $10^{6} \mathrm{~K}$. However, in that model no co-located or co-temporal EBs or UV bursts were reproduced.

Hansteen et al. (2019, Paper I henceforth) takes this analysis further, using a 3D model similar to Hansteen et al. (2017), but starting with a much stronger initial coronal field ( 2 Gauss at $10 \mathrm{Mm}$ ) oriented at a large angle to the direction of the injected emerging field. The initial model used for Paper I is a version of the publicly available "enhanced network" Bifrost model (Carlsson et al. 2016) but where the numerical resolution has been increased by a factor of two, covering the same spatial extent, and with roughly the same magnetic topology. The stronger ambient field helps contain and confine the emerging field, such that large-angle reconnection and powerful coronal heating is produced as the fields interact. The main result of Paper I is that EBs and UV bursts are produced, and occasionally these phenomena are found to be both co-spatial and simultaneous. This result is achieved through forward modeling, synthesizing the $\mathrm{H} \alpha$ and Si IV $139.4 \mathrm{~nm}$ lines, among others. This numerical experiment explains the relationship between EBs and UV bursts as due to a long vertical (or nearly vertical) current sheet that stretches from the photosphere to around $3000 \mathrm{~km}$ upwards where intensive reconnection and heating occurs along the entire current sheet. The EB is formed from the photosphere up to $1200 \mathrm{~km}$ above, while the UV burst is formed at heights between $700 \mathrm{~km}$ and $3 \mathrm{Mm}$ above the photosphere. In other words, these two dynamical phenomena are part of the same system, but occurring at different heights along a reconnection wall.

With respect to the relation of EBs and UV bursts with other phenomena, both have been observed together with surges. The EB-surge relation was reported in the early 1970s (Roy 1973), and has been explored in more recent literature (see, e.g., Watanabe et al. 2011; Vissers et al. 2013; Yang et al. 2013); nonetheless, this relation does not seem to be common (Rutten et al. 2013). A connection has been also established between UV bursts and surges: from the observational point of view (e.g., Guglielmino et al. 2018, 2019) and from a theoretical perspective through numerical experiments (Nóbrega-Siverio et al. 2017 , 2018). Both approaches show that those phenomena occur as a result of magnetic flux emergence from the solar interior. Taking into account the aforementioned findings, it is evident that an in-depth analysis is necessary to scrutinize the temporal 
and spatial relationship between EBs, UV bursts, surges, and magnetic flux emergence.

Here we present multi-wavelength, high-cadence, highresolution coordinated observations to study the possible relationship between reconnection events at different layers in the atmosphere, and in particular, the timing history between them. Our main goal is to determine whether or not there is a temporal and spatial relationship between the appearance of an EB and the appearance of its associated UV burst. Of particular interest is the connection of these transient phenomena to the ejection of surges in the chromosphere. In addition we performed a detailed investigation of the properties of one of the presented UV bursts, carefully examining the variations of these properties with position across the burst area.

The layout of the paper is as follows: Sect. 2 introduces our observations, the data reduction process and identification methods. Section 3 presents our results, which are then discussed and put into context in Sect. 4.

\section{Observations, data reduction, and methodology}

\subsection{Observations and data reduction}

For the work presented here we used coordinated observations between the Interface Region Imaging Spectrograph (IRIS; De Pontieu et al. 2014) and the Swedish $1 \mathrm{~m}$ Solar Telescope (SST; Scharmer et al. 2003a) that were carried out during the last days of August and the beginning of September 2016 centered on AR 12585, which at that time was close to disk center. In particular, we are presenting observations from September 3, 4,5 , and 6 as follows: September 3, center of the IRIS fieldof-view (FOV) $(x, y)=(-561,44)^{\prime \prime}$, heliocentric angle $\mu=0.80$; September 4 , center of the IRIS FOV $(x, y)=(-374,27)^{\prime \prime}$, heliocentric angle $\mu=0.90$; September 5 , center of the IRIS FOV $(x, y)=(-161,24)^{\prime \prime}$, heliocentric angle $\mu=0.98$; and finally September 6, center of the IRIS FOV $(x, y)=(57,22)^{\prime \prime}$, heliocentric angle $\mu=0.99$.

The IRIS data sets correspond to the observing program with OBS-ID 3625503135 carried out every day from 07:44:46 until 10:03:44, or until 10:38:06 UT depending on the day. These observations cover a FOV of $60^{\prime \prime} \times 65^{\prime \prime}$ for the slit-jaw images (SJI) and $5^{\prime \prime} \times 60^{\prime \prime}$ for the spectral raster. This observing program is a medium-dense 16-step raster, which means that a $60^{\prime \prime}$ slit was shifted through 16 positions in the $x$ direction separated by $0 . .33$, with a raster cadence of $20.8 \mathrm{~s}$. The exposure time per slit position was $0.5 \mathrm{~s}$, the IRIS pixel size is 0 '. 166 , and the FUV spectral bins were binned four times in order to increase the signal-to-noise ratio $(\mathrm{S} / \mathrm{N})$. The $\mathrm{SJIs}$ were obtained in three UV bands: at $133 \mathrm{~nm}(F W H M 5.5 \mathrm{~nm}$, dominated by $\mathrm{C}$ II lines and continuum), $140 \mathrm{~nm}$ (FWHM $5.5 \mathrm{~nm}$, dominated by Si IV lines and continuum), and $279.6 \mathrm{~nm}$ ( $F W H M 0.4 \mathrm{~nm}$, centered on $\mathrm{Mg}$ II $\mathrm{k}$ ) with a $10 \mathrm{~s}$ cadence.

With regards to the SST observations, we took spectroscopic images in the $\mathrm{HI} 656.3 \mathrm{~nm}(\mathrm{H} \alpha)$ line, and spectropolarimetric images in the CaII $854.2 \mathrm{~nm}$ line and the Fe I $630.2 \mathrm{~nm}$ line with the CRisp Imaging SPectropolarimeter (CRISP; Scharmer 2006). CRISP is a dual-etalon Fabry-Pérot interferometer mounted in telecentric configuration at the SST. Spectral profiles can be constructed for each pixel over the entire FOV by sequentially acquiring images over a given spectral window, typically at a rate of four spectral positions per second (or one spectral position in polarimetric mode). For this occasion $\mathrm{H} \alpha$ was sampled at 15 spectral positions, from $-0.15 \mathrm{~nm}$ to $0.15 \mathrm{~nm}$ from the line center, with eight exposures per wavelength position.
The Ca II $854.2 \mathrm{~nm}$ line was nonuniformly sampled at 21 positions in polarimetric mode, from $-0.175 \mathrm{~nm}$ to $0.175 \mathrm{~nm}$, taking six exposures per polarimetric state per wavelength position. Finally, the Fe I $630.2 \mathrm{~nm}$ lines were sampled at 16 spectral positions in polarimetric mode with six exposures per polarimetric state per wavelength position. We usually performed short scans that contained only the $\mathrm{H} \alpha$ and Ca II $854.2 \mathrm{~nm}$ lines, at a cadence of $20.2 \mathrm{~s}$. Longer scans contained, in addition to the previous two, the Fe I $630.2 \mathrm{~nm}$ lines at a cadence of $32.2 \mathrm{~s}$. The SST pixel scale at $854.2 \mathrm{~nm}$ is $00^{\prime \prime} 057$ and the FOV was $58^{\prime \prime} \times 58^{\prime \prime}$.

The SST observations were reduced using the CRISPRED reduction pipeline (de la Cruz Rodríguez et al. 2015a) which includes image reconstruction with the Multi-Object-MultiFrame-Blind-Deconvolution technique (MOMFBD, van Noort et al. 2005). The seeing was moderate to good and the image quality benefited from the SST high-order adaptive optics system (Scharmer et al. 2003b).

Aside from using the SST intensity narrowband filtergrams as such, we also constructed photospheric magnetograms from the Ca II $854.2 \mathrm{~nm}$ observations. The formation height range of this line is quite large: while the core samples the midchromosphere, the far wings sample the photosphere, and we can add the Stokes $V$ maps corresponding to the three outer spectral positions in both wings to obtain signed circular polarization maps that serve as a proxy for the photospheric magnetic field (see, e.g., Ortiz et al. 2014).

The temporal overlap between the IRIS and the SST data sets has the following durations: $2 \mathrm{~h}$ and $14 \mathrm{~min}$ (September 3, from 07:49 to 10:03 UT), $1 \mathrm{~h}$ and $9 \mathrm{~min}$ (September 4, from 08:10 to 09:19 UT), $1 \mathrm{~h}$ and $58 \mathrm{~min}$ (September 5, from 07:45 to $09: 44$ UT) and 33 minutes (September 6, from 07:47 to 08:20 UT). The alignment between the CRISP data and the IRIS observations was done in the following way: CRISP data was scaled down to the IRIS pixel scale $\left(0^{\prime \prime} .166\right)$, followed by a cross-correlation of Ca II $854.2 \mathrm{~nm}$ wing images with the SJI $279.6 \mathrm{~nm}$ channel. Table 1 presents a quick overview of our observations. More details about the studied cases are given in Table 2.

To investigate any possible lower chromospheric or coronal response to the reconnection events presented here, we also made use of SDO/AIA imagery (Lemen et al. 2012) from the $170 \mathrm{~nm}$ (temperature minimum, $\sim 5 \times 10^{3} \mathrm{~K}$ ), $30.4 \mathrm{~nm}$ (chromosphere, transition region, $5 \times 10^{4} \mathrm{~K}$ ), $17.1 \mathrm{~nm}$ (upper transition region, $\sim 6.3 \times 10^{5} \mathrm{~K}$ ), and $19.3 \mathrm{~nm}$ (corona, $3 \times 10^{6} \mathrm{~K}$ ) channels, as well as of photospheric magnetograms provided by the Helioseismic and Magnetic Imager (HMI) on board SDO (Scherrer et al. 2012).

\subsection{Ellerman bomb identification in $\mathrm{H} \alpha$ spectra and filtergrams}

For the identification of our EBs, we used the SST blue wing $\mathrm{H} \alpha$ filtergrams at $-0.1 \mathrm{~nm}$ from line center. We made extensive use of the widget-based tool CRISPEX (Vissers \& Rouppe van der Voort 2012) in order to identify the EBs. Once a bright patch was visually identified in the $\mathrm{H} \alpha$ wing filtergram we followed a similar approach to Vissers et al. (2013) to confirm whether the specific brightening was an actual EB or not. We then looked at the $\mathrm{H} \alpha$ profiles of those bright pixels and used an intensity threshold when inspecting their spectra, so that pixels whose wings (both the blue and the red wing) were $150 \%$ or more of the average intensity over the whole FOV were selected. In addition, the core of the line should not present any increase in intensity. After these requirements were imposed, we confirmed (when 
Table 1. Overview of the analyzed data sets.

\begin{tabular}{|c|c|c|c|c|c|}
\hline Case & Observation date & $\begin{array}{l}\text { Coordinates } \\
(x, y)^{\prime \prime}\end{array}$ & $\mu$ angle & $\begin{array}{l}\text { Start time } \\
(\mathrm{UT})\end{array}$ & $\begin{array}{l}\text { Duration of data set } \\
(\mathrm{min})\end{array}$ \\
\hline 1 & $3 / 9 / 2016$ & $\begin{array}{l}(x, y)_{E B}=(-555.7,47.7)^{\prime \prime} \\
(x, y)_{U V}=(-556.5,47.9)^{\prime \prime}\end{array}$ & $\mu=0.80$ & $\begin{array}{l}t_{E B}=08: 13: 30,09: 25: 30 \\
t_{U V}=08: 14: 30,09: 27: 30\end{array}$ & 126 \\
\hline 2 & 6/9/2016 & $\begin{array}{l}(x, y)_{E B}=(58.3,8.1)^{\prime \prime} \\
(x, y)_{U V}=(57.7,8.6)^{\prime \prime}\end{array}$ & $\mu=0.99$ & $\begin{array}{l}t_{E B}=07: 59: 17 \\
t_{U V}=08: 05: 00\end{array}$ & 21 \\
\hline 3 & $5 / 9 / 2016$ & $\begin{array}{l}(x, y)_{E B}=(-162.5,39.5)^{\prime \prime} \\
(x, y)_{U V}=(-162.5,39.8)^{\prime \prime}\end{array}$ & $\mu=0.98$ & $\begin{array}{l}t_{E B}=09: 26: 04 \\
t_{U V}=09: 26: 36\end{array}$ & 23 \\
\hline 4 & $5 / 9 / 2016$ & $(x, y)_{E B}=(-158.0,3.5)^{\prime \prime}$ & $\mu=0.98$ & $t_{E B}=08: 16: 36$ & 17 \\
\hline 5 & $3 / 9 / 2016$ & $\begin{array}{l}(x, y)_{E B}=(-563.4,25.5)^{\prime \prime} \\
(x, y)_{U V}=(-562.9,25.5)^{\prime \prime}\end{array}$ & $\mu=0.80$ & $\begin{array}{l}t_{E B}=08: 50: 48,08: 58: 13 \\
t_{U V}=08: 51: 08,08: 59: 34\end{array}$ & 68 \\
\hline 6 & $4 / 9 / 2016$ & $(x, y)_{U V}=(-369.3,41.0)^{\prime \prime}$ & $\mu=0.90$ & $t_{U V}=08: 10: 46,08: 58: 59$ & 69 \\
\hline 7 & $5 / 9 / 2016$ & $(x, y)_{U V}=(-160.7,37.1)^{\prime \prime}$ & $\mu=0.98$ & $t_{U V}=08: 53: 00$ & 29 \\
\hline 8 & $5 / 9 / 2016$ & $(x, y)_{U V}=(-160.3,10.8)^{\prime \prime}$ & $\mu=0.98$ & $t_{U V}=09: 08: 53,09: 15: 20$ & 29 \\
\hline
\end{tabular}

Notes. Columns are as follows: observation date, coordinates of the reconnection event in arcsec, heliocentric angle, starting time of each reconnection event (UT, separated by comma if multiple brightenings), total duration of data set in minutes.

Table 2. Characteristics of the eight transient brightenings presented in this work.

\begin{tabular}{|c|c|c|c|c|c|}
\hline Case & IRIS slit & $\begin{array}{c}\text { Type of } \\
\text { brightening }\end{array}$ & $\begin{array}{c}\text { Duration } \\
(\mathrm{min})\end{array}$ & $\begin{array}{c}\text { Delay of UV } \\
\text { burst (min) }\end{array}$ & Surge \\
\hline 1 & No & $\mathrm{EB}+\mathrm{UV}$ burst & $31+38^{(a)}, 25.5+36^{(b)}$ & 1 & Yes \\
\hline 2 & Yes & $\mathrm{EB}+\mathrm{UV}$ burst & 21,15 & 6 & Yes \\
\hline 3 & Yes & $\mathrm{EB}+\mathrm{UV}$ burst & $22,7.5$ & 0.5 & Yes \\
\hline 4 & Yes & $\mathrm{EB}$ & 9 & - & no \\
\hline 5 & Yes & $\mathrm{EB}+\mathrm{UV}$ burst & $6,4.5^{(c)}$ & 0.5 & Yes \\
\hline 6 & No & UV burst & $47+19^{(b)}$ & - & Yes \\
\hline 7 & Yes & UV burst & $6+7+1^{(b)}$ & - & Yes \\
\hline 8 & Yes & UV burst & $7+6+2^{(b)}$ & - & Yes \\
\hline Case & $\begin{array}{c}\text { Mg II triplet } \\
\text { emission }\end{array}$ & $\begin{array}{l}\text { Mn I absorption on } \\
\text { Mg II k blue wing }\end{array}$ & $\begin{array}{l}\text { Ni II absorption } \\
\text { on Si IV } 139.4 \mathrm{~nm}\end{array}$ & $\begin{array}{c}\text { Coronal response } \\
\text { in AIA } \\
\end{array}$ & $\begin{array}{l}\text { AIA } 170 \mathrm{~nm} \\
\text { brightening }\end{array}$ \\
\hline 1 & - & - & - & No & yes \\
\hline 2 & Yes & Yes $^{(d)}$ & Yes & No & Yes \\
\hline 3 & Yes & Yes $^{(d)}$ (weak) & Yes & No & Yes \\
\hline 4 & No & No & No & - & - \\
\hline 5 & Yes (weak) & No & No & No & Yes \\
\hline 6 & - & - & - & Yes & Yes \\
\hline 7 & No & Yes (very weak) & No & No & Yes \\
\hline 8 & No & No & Yes & No (Yes in $30.4 \mathrm{~nm}$ ) & No \\
\hline
\end{tabular}

Notes. Columns are as follows: event happens at the slit positions of the IRIS spectrograph, type of brightening, duration of each reconnection event (in minutes, first number refers to the EB, second number to the Si IV UV burst, separated by a comma), delay of the UV burst with respect to the EB (in minutes, only for cases where both co-exist), presence of an associated surge, existence of $\mathrm{Mg}$ II triplet in emission, existence of Mn I absorption lines superimposed on the blue wing of Mg II k profiles, existence of Ni II absorption lines superimposed on the Si IV $139.4 \mathrm{~nm}$ profiles, coronal response in the AIA channels, and brightening in the AIA $170 \mathrm{~nm}$ channel. ${ }^{(a)}$ This EB presents several brightenings. These numbers refer to each brightening. ${ }^{(b)}$ This UV burst presents several brightenings. These numbers refer to each brightening. ${ }^{(c)}$ These events present several intensity peaks. These values refer to the peaks with higher intensity. ${ }^{(d)}$ During some time steps.

inspecting the photospheric magnetograms in the Ca II $854.2 \mathrm{~nm}$ line) that the selected EB locations were indeed sites of merging between magnetic fields of opposite polarities. This procedure gave us the initial location of the EB, which was chosen as the pixel with highest intensity within the bright patch complying with the mentioned requirements.

A $15^{\prime \prime} \times 15^{\prime \prime}$ box was then selected around the initial position of the $\mathrm{EB}$ in the $2 \mathrm{D} \mathrm{H} \alpha$ wing images. For the subsequent time steps, the location of the EB was automatically inferred by calculating the centroid of those pixels inside the box above a certain intensity threshold. This threshold was set to 6500 counts in the $\mathrm{H} \alpha$ wing filtergrams. If no pixel was above the threshold at a particular time step, the position of the EB was taken as the position in the previous time step. The intensity given for the EB at a given time was calculated as the average of the fifty brightest pixels inside the box. This procedure therefore avoids the consideration of pixels inside the box that did not have an intensity above the minimum set and thus were not part of the EB. The $\mathrm{H} \alpha$ and Ca II light curves presented in Figs. 2, 9, 11, and 14 follow this method. Movies of $\mathrm{H} \alpha-0.1 \mathrm{~nm}$ showing the temporal evolution and trajectories of the selected EBs are available online. 


\subsection{Ultraviolet burst identification in IRIS SJIs and spectra}

For the identification of UV bursts, we used the Si IV $140 \mathrm{~nm}$ SJIs and spectra, following the definition of Peter et al. (2014). In those SJIs, we identified small $\left(\sim 1^{\prime \prime}\right)$, compact, roundish transient brightenings with substantial emission in the Si IV spectral lines observed by IRIS, whose spectra show very wide and complex nonGaussian profiles on which deep absorption blends of lower metal ionization stages are superimposed. This definition gave us the initial location of the UV burst. Similar to the EB identification, we chose the pixel within the UV patch that presented a higher Si IV intensity.

Again a $15^{\prime \prime} \times 15^{\prime \prime}$ box was then selected around the initial position of the burst in the Si IV $140 \mathrm{~nm}$ SJIs. Automatic detection of the UV burst in subsequent time steps was carried out in the same way as for EBs, that is by calculating the centroid of pixels inside the box above a certain intensity threshold, which in this case was set to 12 counts for the $140 \mathrm{~nm}$ SJIs. The intensity of the UV burst at a given time step was taken as the average of the fifty brightest pixels inside the box. Whenever there was a South Atlantic Anomaly (SAA) event, the location of the UV burst was frozen at the position of the last time step not affected by the SAA in order to avoid any spurious jumping of our detection box. The FUV and NUV light curves in Figs. 2, 9, 11, and 14 follow this method. Movies of Si IV $140 \mathrm{~nm}$ and $\mathrm{Mg}$ II k $279.6 \mathrm{~nm}$ SJIs showing the temporal evolution and trajectories of the identified UV bursts are available online.

\subsection{Time-sliced spectra}

As part of the spectral analysis presented here we plot the spectra of EBs and UV bursts as a function of time (time-sliced spectra). The purpose is to show the temporal evolution of their spectra. The spectra shown for each time step is that of the centroid calculated in Sects. 2.2 and 2.3 at that given time step, that is, we follow the trajectory of the EB or UV burst as previously explained and show its spectra at every given time step. Since coexisting EBs and UV bursts follow different trajectories (albeit closely related), the spectra shown in the time-sliced spectra plots for EBs are not derived at exactly the same pixel as its associated UV burst. In the case where only an EB was present with no associated UV brightening, both the SST and the IRIS spectra have been plotted at the same pixel, that is, the one given by the trajectory of the EB. The same was done for the case of a UV burst only.

\section{Results}

We analyzed a total of eight cases of transient brightenings associated with magnetic reconnection in the flux emergence region present within AR 12585, for the period September 3-6, 2016. The criteria for selecting those eight cases was that most of them should be located within the slit positions of the IRIS spectrograph, so that UV spectra - and not just visible or infrared spectra from the SST - were also available. Six out of the eight cases lie within the FOV of the IRIS slit spectrograph. The remaining two cases do not fall within the IRIS slits, but we chose them for their particular interest (both are long-lived brightenings).

Four of the eight studied cases are presented in more detail in the sections below as they are representative of the whole sample, and they are moreover covered by the IRIS raster.

Table 2 summarizes the characteristics of the eight cases of transient brightenings studied here. It is worth noting that some of the cases presented here have been examined for other purposes in the literature. Case 1 was analyzed by NóbregaSiverio et al. (2017), Rouppe van der Voort et al. (2017) and Vissers et al. (2019), while Rouppe van der Voort et al. (2017) and Vissers et al. (2019) focus part of their study on time steps neighboring our case 3 .

\subsection{Coexistence of an EB and a UV burst: September 6 , 2016}

On 6 September 2016, between 07:59 and 08:20 UT, an EB and a UV burst coexisted spatially and temporally. The initial position of the EB was $(x, y)=(58.3,8.1)^{\prime \prime}$ at 07:59:17 UT, while the initial position of the UV burst was $(x, y)=(57.7,8.6)^{\prime \prime}$ starting at 08:05:00 UT. This pair of brightenings is presented in Fig. 1, which shows a mosaic of images from the SST, IRIS, and SDO observatories at 08:12 UT (except for the SDO imagery, which is taken at 08:05:21 UT). This FOV is the site of vigorous flux emergence happening southeast of the sunspot partially seen in the upper-right part of the panels.

The location where the brightenings occur is the site of merging of magnetic fields of opposite polarity, as shown by the Stokes $V$ maps in the wings of Ca II $854.2 \mathrm{~nm}$ and the HMI magnetograms. This is common for the eight cases presented here. The positive and negative polarities seem to interact intermittently.

In the panels of Fig. 1 the EB has been highlighted with a red contour (see Sect. 2.2 for details on the identification process), while the UV burst has been highlighted with a yellow contour (Sect. 2.3 gives details on the process followed to identify UV bursts). The UV burst is evident in both the IRIS SJI $140 \mathrm{~nm}$ and $279.6 \mathrm{~nm}$. In these images the EB reaches a maximum diameter of around $1.5^{\prime \prime}$ with a roundish or elongated shape during its lifetime. The UV burst presents a peak diameter of around 4.5" with a predominantly circular shape. During the time of their co-existence the UV burst lies exactly on top of the EB, with the latter always occupying the center of the UV burst extension.

The H $\alpha$ wing images show a surge shooting out diagonally from the lower-left side of the EB at around 08:06 UT that lasts for $13 \mathrm{~min}$, almost until the end of the observations. The surge is a very dynamical structure with a maximum length of $16^{\prime \prime}$ (reached at 08:14:27 UT) in the $\mathrm{H} \alpha-0.1 \mathrm{~nm}$ images. The surge can also be observed in the Ca II $854.2 \mathrm{~nm}$ panels, both in the wings and the core of the line. The temporal evolution of the surge can be seen in the $\mathrm{H} \alpha$ and $\mathrm{Ca}$ II $854.2 \mathrm{~nm}$ movies available online.

While the AIA $170 \mathrm{~nm}$ panel of Fig. 1 shows a brightening at the common position of the EB and UV burst, the transition region and coronal wavelengths do not show any signature associated to those reconnection events. What these high-temperature EUV diagnostics show instead is indeed a darkening roughly coinciding with the surge. The cold dense material of the surge is opaque due to the presence of neutral hydrogen and helium and absorbs eventual radiation from the reconnection events not allowing any generated radiation in these EUV bands to escape.

Figure 2 presents the temporal evolution of the EB and UV burst intensities at several wavelengths for the duration of their coexistence. The light curves were calculated as detailed in Sects. 2.2 and 2.3. The upper panel shows SST intensities for $\mathrm{H} \alpha-0.1 \mathrm{~nm}, \mathrm{H} \alpha$ line center, Ca II $854.2-0.0735 \mathrm{~nm}$ and line center. Both the $\mathrm{H} \alpha$ intensities in the wing and the core of the line show a decrease at the time when the surge is totally covering the EB or is more active. The middle panel shows the intensity derived from the IRIS SJIs $279.6 \mathrm{~nm}$ images, while the 


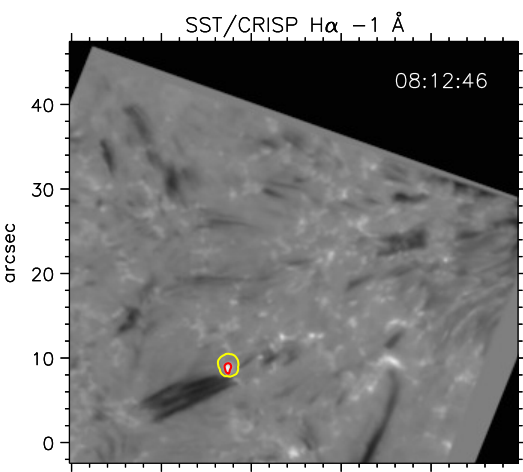

SST/CRISP Co $\| 8542-0.735 \AA$
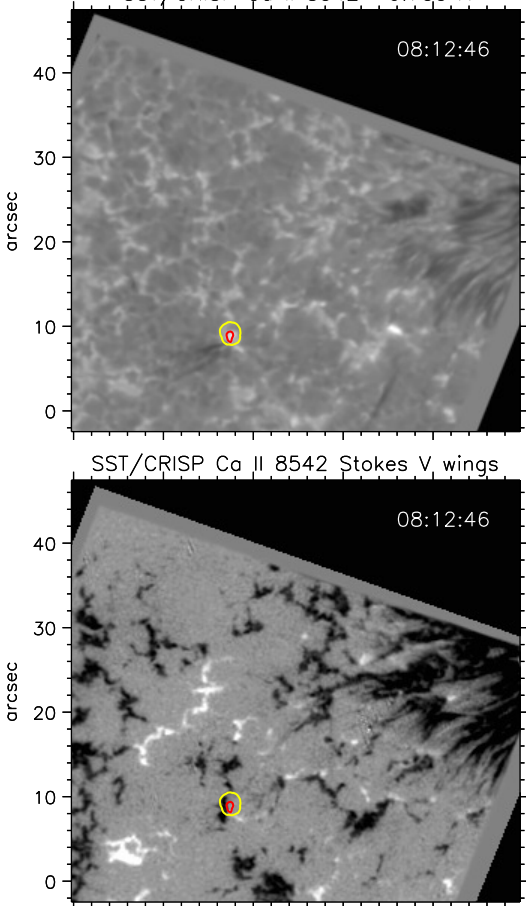

SDO/AIA $304 \AA$

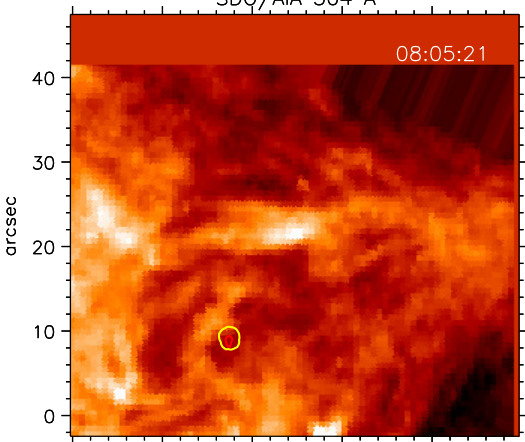

$40 \quad 50$

$60 \quad 70$

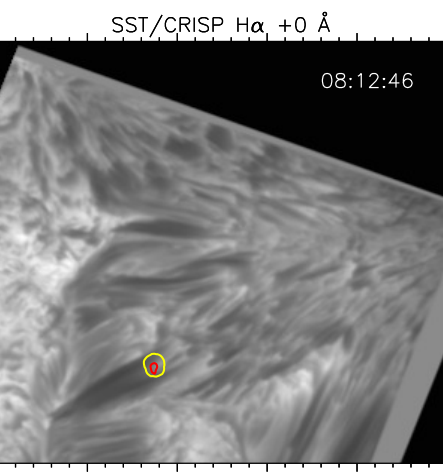

SST/CRISP Ca $\| 8542+0 \AA$

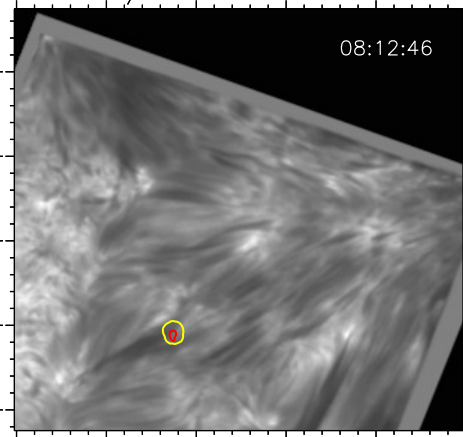

$\mathrm{SDO} / \mathrm{HMI}$ mognetogram

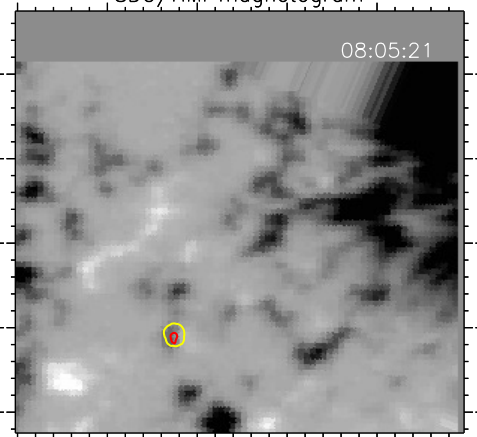

SDO/AIA $171 \AA$

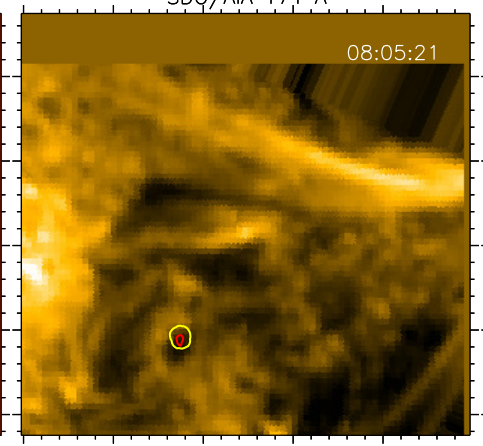

$40 \quad 50 \quad 60$ $70 \quad 80$ 40

$\operatorname{arcsec}$

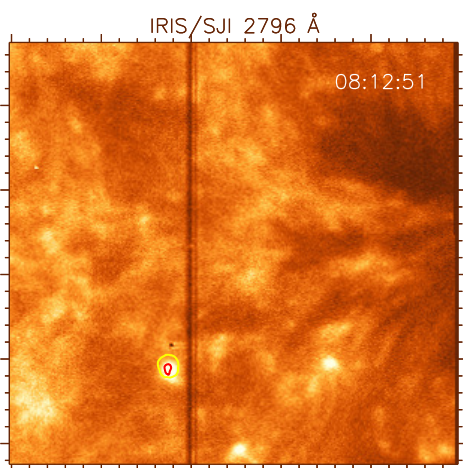

IRIS/SJI $1400 \AA$

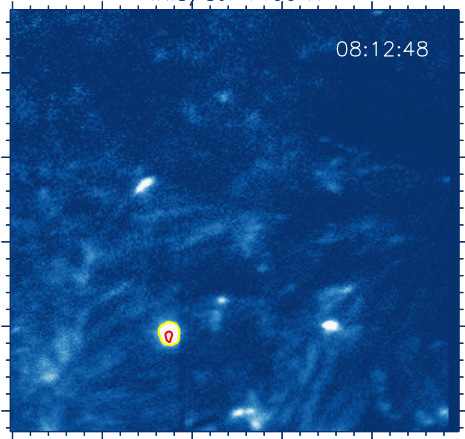

SDO/AIA $1700 \AA$

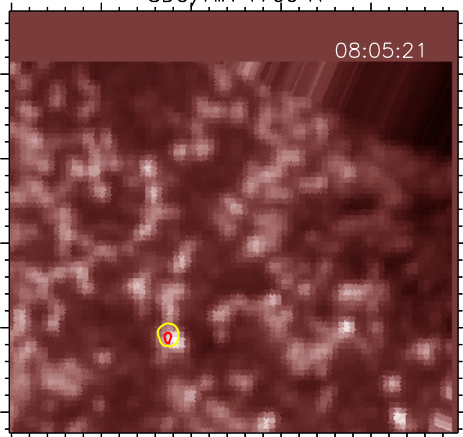

SDO/AIA $193 \AA$

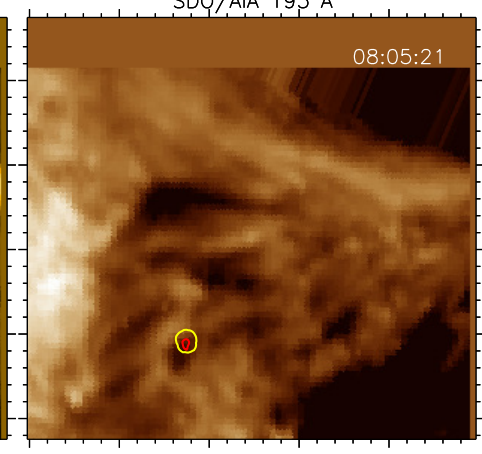

60

Fig. 1. Mosaic of images from SST, IRIS and SDO for September 6, 2016. At this particular time both an EB and a UV burst co-existed temporally and spatially. Upper row (from left to right): SST H $\alpha$ wing at $-0.1 \mathrm{~nm}$, SST H $\alpha$ core, and IRIS SJI $279.6 \mathrm{~nm}$. Second row: SST Ca II $854.2 \mathrm{~nm}$ wing at $-0.0735 \mathrm{~nm}$, SST Ca II $854.2 \mathrm{~nm}$ core and IRIS SJI $140 \mathrm{~nm}$. Third row: SST Stokes $V$ map in the wings of Ca II $854.2 \mathrm{~nm}$, SDO/HMI magnetogram and SDO/AIA $170 \mathrm{~nm}$. Fourth row: SDO/AIA $30.4 \mathrm{~nm}$, SDO/AIA $17.1 \mathrm{~nm}$ and SDO/AIA $19.3 \mathrm{~nm}$. Red and yellow contours in all the panels highlight the location of the EB and UV burst, respectively. The Stokes $V$ map in the wings of Ca II $854.2 \mathrm{~nm}$ and the HMI photospheric magnetogram show that both reconnection events happen at a merger site of opposite magnetic polarities. The AIA/SDO images do not show any coronal counterpart of these reconnection events, but a diminished intensity due to absorption by the surge.

lower panel presents the intensity obtained from the SJI $140 \mathrm{~nm}$ images.

The EB starts at 07:59 UT and is still present at the end of our observations, lasting at least $21 \mathrm{~min}$ (see Table 2). The UV brightening however appears 6 min later, at 08:04:30 UT in the SJI $279.6 \mathrm{~nm}$ and at 08:05:00 UT in the SJI $140 \mathrm{~nm}$ images. At the end of the data set it is still easily visible in both wavelengths, thus existing for at least $15 \mathrm{~min}$. Its intensity peaks at 

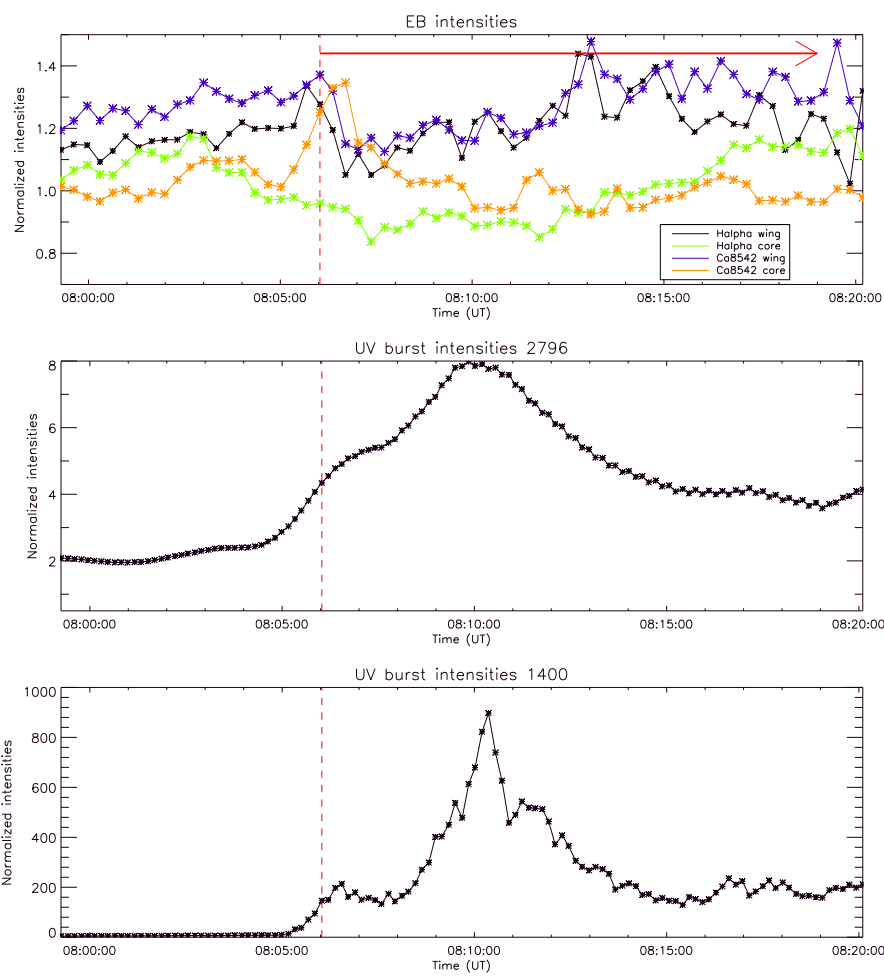

Fig. 2. Intensity as a function of time during the duration of the cospatial and co-temporal EB and UV burst brightenings for September 6, 2016. Upper panel: average SST H $\alpha$ (wing and core, black and green respectively) and Ca II $854.2 \mathrm{~nm}$ (wing and core, blue and orange respectively) intensities in a box enclosing the EB. Middle panel: average IRIS SJI $279.6 \mathrm{~nm}$ intensity in a box enclosing the UV burst. Lower panel: average IRIS SJI $140 \mathrm{~nm}$ intensity in a box enclosing the UV burst. The vertical dashed line and the red arrow mark the onset and lifetime of the surge.

08:10:00 UT in Mg II (middle panel) and 30 s later in Si IV (lower panel). The EB intensity does not show substantial variation (only a maximum at 08:05:41 and 08:12:46 UT), while the UV burst intensity increases impulsively taking only $5 \mathrm{~min}$ from its appearance in the FOV until it reaches its maximum intensity.

Figure 2 reveals a bursty behavior, especially in the Si IV intensity. This is common to the other UV bursts analyzed here. The timescale of this intermittent behavior in our observations is of the order of a few minutes (well sampled by our IRIS observations at a $10 \mathrm{~s}$ cadence), varying between three and ten minutes depending on the case. Similar variations have been reported by Young et al. (2018) and Guglielmino et al. (2018). The light curves of the Mg II SJIs have a slightly smoother behavior when compared with the temporary evolution of the Si IV SJIs, as was also observed by Guglielmino et al. (2018).

Figure 3 presents spectral profiles for different SST and IRIS lines as well as their temporal evolution. In particular, the left panel shows the profiles for $\mathrm{H} \alpha$ and $\mathrm{Ca}$ II $854.2 \mathrm{~nm}$ (top), $\mathrm{Mg}$ II k and $\mathrm{h}$ lines (middle), and the Si IV lines at 139.4 and $140.3 \mathrm{~nm}$, respectively (bottom), for four selected time steps. These time steps are representative of the beginning of the EB (07:59 UT, black line, start of the observations), the beginning of the UV burst (08:05 UT, blue line), the peak of the UV burst diameter (08:12 UT, green line), and finally the end of the observations (08:20 UT, red line). The panel on the right shows a time-sliced spectrum showing the temporal evolution of three spectral lines: SST H $\alpha$, IRIS Mg II $\mathrm{k}$ and triplet lines and IRIS Si IV $139.4 \mathrm{~nm}$. We have followed the procedure detailed in
Sect. 2.4. Red and blue arrows in the time-sliced spectrum pinpoint specific moments in the evolution of the brightenings. The lower red arrow marks the beginning of the EB and the observations themselves. The middle red arrow points to the beginning of the UV burst. The top red arrow highlights the moment of maximum diameter of the UV burst. The blue arrows (08:06:45 to $08: 07: 30 \mathrm{UT}$ ) enclose the time of maximum $\mathrm{H} \alpha$ intensity drop due to the passage of the surge hiding the EB from the view of the observer (this can also be clearly seen in the $\mathrm{H} \alpha$ movies for September 6, 2016, available online).

As shown by the top left panel of Fig. 3, the EB is brighter (i.e., has a higher increase in the $\mathrm{H} \alpha$ wings) at around 08:05 UT right before the surge covers the EB - with a similar behavior in the wings of CaII $854.2 \mathrm{~nm}$. After the intensity decrease at 08:06:45 UT in the wings of $\mathrm{H} \alpha$, a recovery of the EB intensity follows until 08:13 UT. The UV burst on the other hand reaches a peak in diameter and brightness between 08:09 and 08:13 UT. This is evident in the $\mathrm{Mg}$ II $\mathrm{k}$ and $\mathrm{h}$ profiles at $279.6 \mathrm{~nm}$ and the Si IV profiles at 139.4 and $140.3 \mathrm{~nm}$ at $08: 12 \mathrm{UT}$ in the left panels of Fig. 3 (green line). In the case of the Si IV profiles the intensity increases by one order of magnitude when compared to any other time steps. In the case of the Mg II profiles, the amplitude of the $\mathrm{k} 2 \mathrm{v}$ and $\mathrm{k} 2 \mathrm{r}$ peaks doubles at 08:12 UT relative to the other times.

Inspection of the $\mathrm{Mg} \mathrm{II} \mathrm{k}$ and $\mathrm{h}$ profiles, as well as the timesliced spectrum, reveals the presence of the Mg II triplet in emission for about 9 min around the time of maximum diameter and brightness of the UV burst. According to Pereira et al. (2015), the triplet comes into emission after a temperature increase in the lower chromosphere of more than $1500 \mathrm{~K}$. The Mg II triplet in emission is very evident in the center-left panel of Fig. 3 at 08:12 UT. The presence of the Ni II absorption line is also evident when inspecting the Si IV $139.4 \mathrm{~nm}$ profiles (left panel) and time-sliced spectra (right panel). The deep superimposed on its left wing is well visible in the Si IV profile at 08:12 UT (green line) due to the increased intensity of that profile. However, the time-sliced spectrum reveals that the absorption is present for the whole duration of the burst at that particular point and not only at the time of maximum brightness.

\subsection{A closer view of the UV burst: properties}

In this section we analyze in more detail the properties of the UV burst and its variations with position within the burst area.

This UV burst has an approximate extension of $2^{\prime \prime} \times 2^{\prime \prime}-$ reaching almost $2^{\prime \prime} \times 3^{\prime \prime}$ in its period of maximum extension when seen in the spectral rasters of IRIS. It has a roundish shape when observed in the $140 \mathrm{~nm}$ IRIS SJI (which is maintained throughout its lifetime) but appears slightly smaller when seen in the spectrograph rasters. The Si IV $140.3 \mathrm{~nm}$ profiles observed within the UV burst area come in a variety of shapes: clearly double-peaked, clearly singled-peaked, or a mixture of both, that is, mainly single-peaked with a lesser second component in one of the wings.

In Fig. 4 we present the velocity maps derived from Si IV $140.3 \mathrm{~nm}$ profiles corresponding to three time steps around the maximum peak of intensity of the UV burst. The moment of maximum intensity of the UV burst can be seen in the middle and lower panels of Fig. 2. In particular we have chosen the following times: before the maximum at 08:09:05 UT, during the maximum at 08:10:08 UT, and after the maximum at 08:12:13 UT. The black contours delimit the boundaries of the UV burst by imposing a threshold of 20 counts to the Si IV raster intensities. The velocities represented in Fig. 4 have been derived 

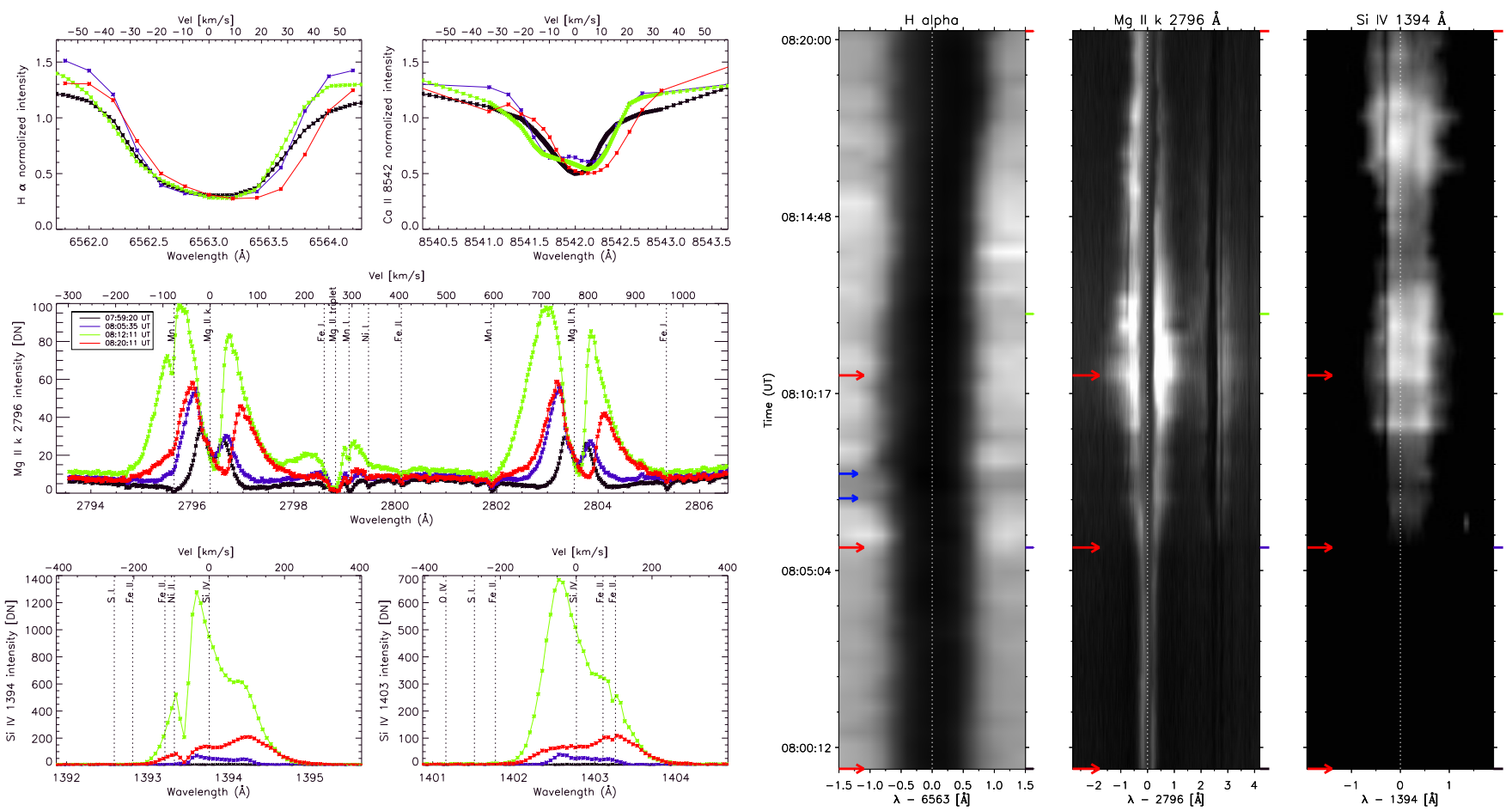

Fig. 3. Left panel: spectra of different lines for September 6, 2016, at specific times (see legend) during the evolution of the EB and the UV burst. Upper row: H I $656.3 \mathrm{~nm}$ and Ca II $854.2 \mathrm{~nm}$ respectively; mid row: $\mathrm{Mg}$ II $\mathrm{k}$ and h lines at $279.6 \mathrm{~nm}$; lower row: Si IV lines at 139.4 and $140.3 \mathrm{~nm}$ respectively. The wavelengths at rest of some reference lines are indicated by vertical dotted lines. Right panel: time-sliced spectrum, also for September 6 2016, for H I $656.3 \mathrm{~nm}$ (left), Mg II k and triplet lines (center) and the Si IV $139.4 \mathrm{~nm}$ line (right). The Ni II absorption line can be seen superimposed on the left wing of Si IV $139.4 \mathrm{~nm}$ during the whole duration of the UV brightening. Red and blue arrows mark specific moments in the evolution of the brightenings (see text for details). The colored marks pinpoint to the same times that have been highlighted in the left panel. We have applied a gamma adjustment to the Si IV color table.

by fitting a single Gaussian to the Si IV profiles. While several profiles within the burst area are double-peaked as mentioned before - and therefore could be very well fit using a double Gaussian - the same double Gaussian fit works extremely poorly for those pixels that are single-peaked or a hybrid between single and double peaks. We therefore opted for using a single Gaussian fit throughout the whole UV burst domain in order to construct the maps shown in Fig. 4 at the expense of obtaining more conservative velocities in those pixels which show double-peaked profiles (thus not properly represented by a single Gaussian fit). The maximum velocities yielded by this single fit method are $\pm 50 \mathrm{~km} \mathrm{~s}^{-1}$ (with the exception of a few pixels yielding $-75 \mathrm{~km} \mathrm{~s}^{-1}$ ). The pixels outside of the UV brightening have a very small signal-to-noise ratio and therefore no function can be properly fitted. Only pixels with peak intensity above $2.5 \sigma$ have been included in the fits.

Realizing that the velocities yielded by the fit of a single Gaussian are quite conservative in many of the burst pixels (especially in the double-peaked ones), we have individually fitted a double Gaussian to some of those pixels which are more accurately represented by this function. In this case the velocities reach up to $-50 \mathrm{~km} \mathrm{~s}^{-1}$ for the blueshifted component and up to $100 \mathrm{~km} \mathrm{~s}^{-1}$ for the redshifted component, both at 08:09:05 and 08:10:08 UT. Such high values are not reached at 08:12:13 UT as this time step shows lower velocities.

We note that the burst domain is clearly divided into three different zones (redshifted velocities, blueshifted velocities, and zero velocity) at all times, as the observed Si IV profiles in those zones are redshifted, blueshifted, or at rest respectively. The redshifted region is centered around $57^{\prime \prime}$ in the $x$-direction with a width of roughly $1^{\prime \prime}$, while stretching some $2^{\prime \prime}$ in the $y$-direction from $7.2^{\prime \prime}$ to $9.2^{\prime \prime}$. The blueshifted region lies $1^{\prime \prime}$ to the right with a width of slightly less than $1^{\prime \prime}$. Initially the extent of the blueshifted region is slightly longer than the redshifted region, stretching from $6.8^{\prime \prime}$ to $9.2^{\prime \prime}$, but at later times the red- and blueshifted regions are of very similar extent. The Si IV velocities are highest before and during the peak of maximum intensity, but decrease significantly at the time of the last panel at 08:12:13 UT.

Representative Si IV profiles for each of the above-mentioned zones are given in Fig. 5. This figure shows the Si IV profiles corresponding to those pixels marked by a cross in the central panel of Fig. 4 and showcases the variety of shapes found across the burst domain. The red curve is a double-peaked redshifted profile and corresponds to the pixel marked by a cross in the redshifted zone of the UV brightening. The blue curve is a blueshifted single-peaked profile with a smaller component in its red wing, and corresponds to the pixel marked by a cross in the blueshifted region of the UV burst. Finally, the black curve is a single-peaked profile centered at the rest wavelength of Si IV $140.3 \mathrm{~nm}$ and belongs to the pixel marked by a cross in the white (zero velocity) region of the burst.

In the redshifted zones of Fig. 4 most of the pixels (79\%) have double-peaked profiles, while the remaining $21 \%$ are single-peaked Si IV profiles. These proportions change in the blueshifted zones of the UV burst, which are composed of $3 \%$ with double-peaked profiles, $65 \%$ with single-peaked profiles, and $32 \%$ with single-peaked profiles that contain a lesser second component in one wing. Finally, the white areas of the velocity maps, that is, where the Si IV profiles are mainly at rest, are $100 \%$ 


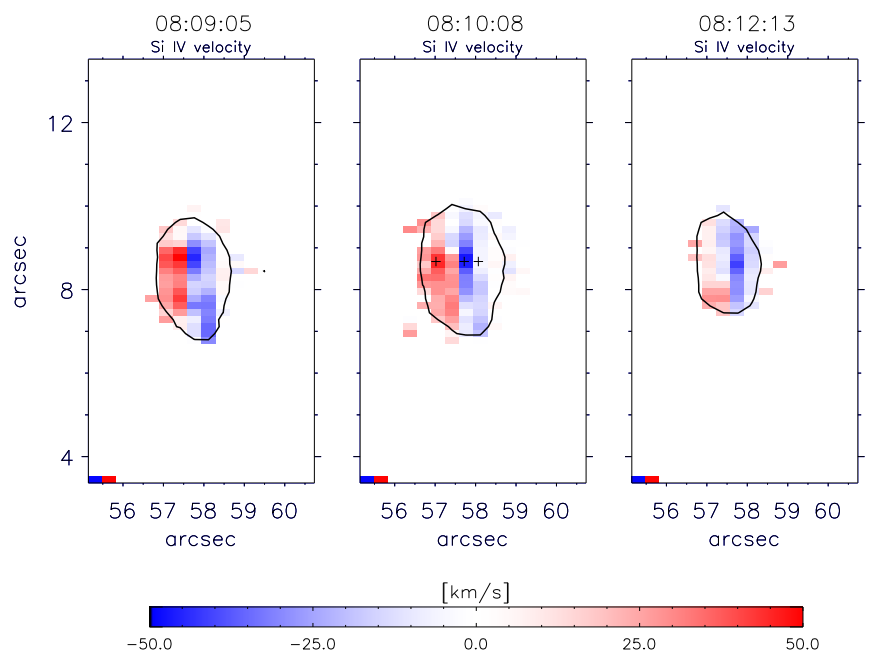

Fig. 4. Maps of the Si IV $140.3 \mathrm{~nm}$ velocity for three time steps: from left to right, 08:09:05, 08:10:08 and 08:12:13 UT. The time steps have been chosen around (before, during, and after) the maximum intensity peak of the burst. The time of maximum intensity can be found in Fig. 2 slightly after 08:10 UT. A single Gaussian has been fitted to the Si IV $140.3 \mathrm{~nm}$ profiles (but see text for additional explanations). The crosses in the central panel mark the positions of the pixels whose profiles are shown in Fig. 5. The black contours delimit the boundaries of the UV burst for the different time steps by imposing a threshold to the Si IV intensity.

populated by single-peaked profiles. In this particular case, the majority of the parts of the UV burst that are closer to disk center show double-peaked redshifted profiles, while the parts further away from disk center present mostly blueshifted (or at rest) single-peaked profiles.

Double-peaked Si IV profiles are often interpreted as bidirectional jets expelled from the reconnection site both towards and away from the observer. Singled-peaked profiles with a smaller satellite component in either wing could also be interpreted as the observation of plasma ejected in one direction as a result of reconnection, plus another jet with a smaller Dopplershift ejected in the opposite direction in the line of sight. Overall, the structure of the observed velocities and intensities is suggestive of a nearly vertical current sheet, oriented in the $y$-direction but slightly slanted with respect to the vertical. Reconnection will occur along such a current sheet as supported by Paper I, producing irregular bidirectional jets and in this case producing mainly downflows around $x=57^{\prime \prime}$ and mainly upflows around $x=58^{\prime \prime}$. The fact that profiles closer to disk center are mostly doublepeaked while those further away are mostly single-peaked with a satellite component is most likely due to projection effects. In parts of the burst pointing away from the observer where bidirectional jets are in place, one component may be damped with respect to the other.

Figure 6 shows intensity maps corresponding to IRIS rasters centered at the nominal wavelengths of the following line features (from left to right): $\mathrm{Mg}$ II $279.6 \mathrm{~nm} \mathrm{k} 3, \mathrm{k} 2 \mathrm{v}, \mathrm{k} 2 \mathrm{r}$, triplet and Si IV $140.3 \mathrm{~nm}$. The Si IV maps show the intensity integrated over the whole line profile, unlike the Mg II maps which are centered at a particular wavelength. We have selected the same time steps around the maximum peak of intensity of the UV burst as in Fig. 4. The solid orange contours delimit the UV burst as seen in the Si IV intensity, while the dashed orange contours mark the boundaries of the burst as seen in the $\mathrm{Mg}$ II triplet line feature. We note that the UV brightening is not visible at all wavelengths

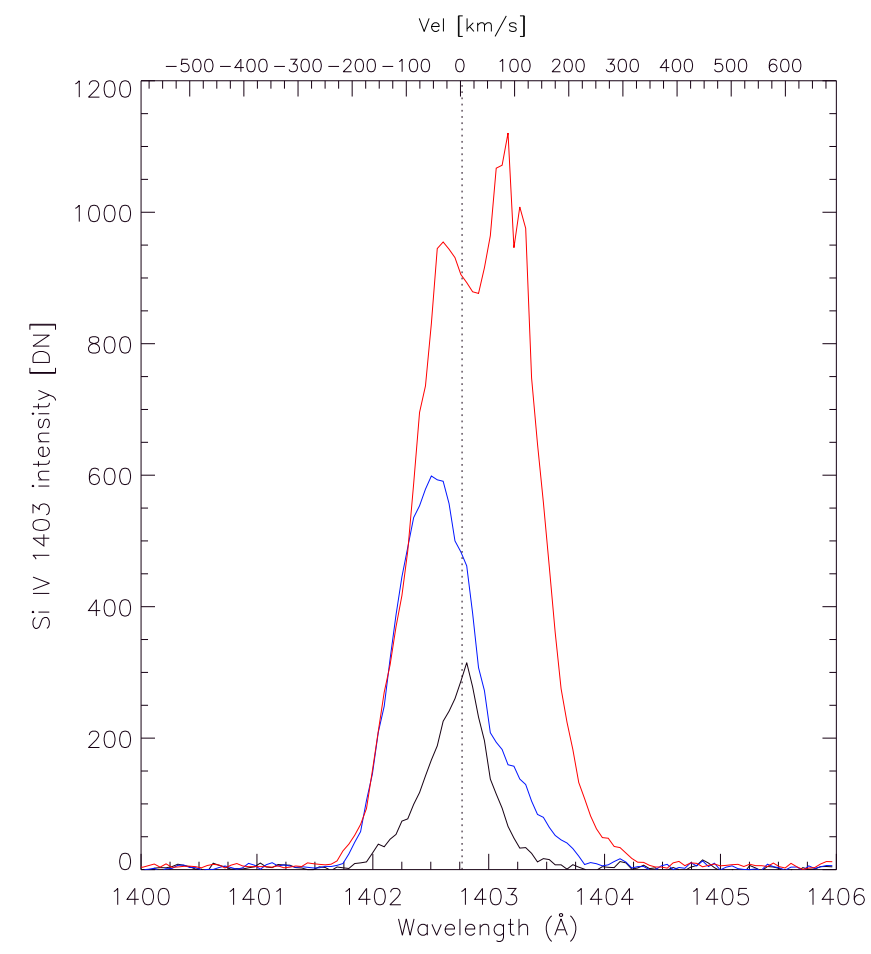

Fig. 5. Si IV $140.3 \mathrm{~nm}$ profiles corresponding to the locations marked by a cross in Fig. 4 at 08:10:08 UT (central panel). The red curve corresponds to the pixel marked by a cross within the red patch of the burst, the blue curve corresponds to the pixel marked by a cross within the blue patch of the burst, and the black curve corresponds to the pixel highlighted with a cross within the white patch of the burst in the central panel of Fig. 4.

due to a canopy of overlying cold fibrils, and therefore the contours have been derived from those wavelengths where the burst is always visible.

Within the spectral rasters, the morphology of the burst changes between the Mg II and Si IV maps: the UV brightening appears more slender in the FUV than in the NUV. This may be related to what is found in numerical experiments. In the simulations of Paper I (see e.g. Fig. 1), the magnetic field expands with height so the reconnecting current sheet grows gradually wider, but in the direction perpendicular to the current sheet the visible width of the current sheet becomes thinner. This is a result of the plasma- $\beta$ (the ratio of gas pressure to magnetic pressure) decreasing with height: the magnetic field and the gas are more or less equally matched in the upper photosphere leading to rounder more amorphous structures there as the current sheet is deformed by plasma motions, but at greater heights the magnetic field completely dominates, the field lines straighten out and fill all space, and the current sheet delineates two regions of equally strong straight field that are pointing in different (opposite) directions. Even within the same time step of Fig. 6 the intensity maps in the different $\mathrm{Mg}$ II features differ significantly from each other, as well as from the associated Si IV map. In the $\mathrm{k} 3$ core we do not see any evidence of the UV burst at all at any time. A similar situation is observed in the Mg II $\mathrm{k} 2 \mathrm{r}$ peak, where only at 08:10:08 UT can a slight hint of the UV brightening be seen. This is because diagonally oriented fibrils of cold material apparently cover the burst at both of these wavelengths. These cold fibrils are most likely related to the surge, as they follow the same diagonal direction and this is the period around which the surge reaches its maximum length and shows very dynamic 

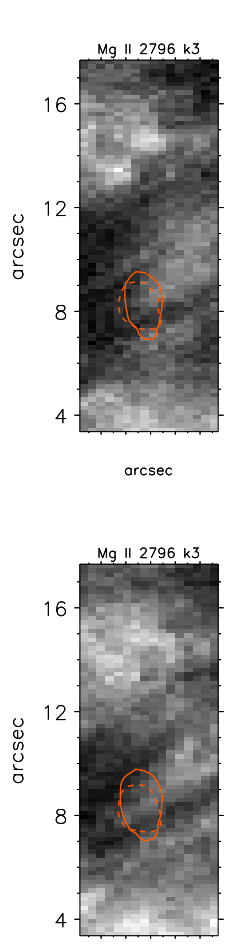

$\operatorname{arcsec}$

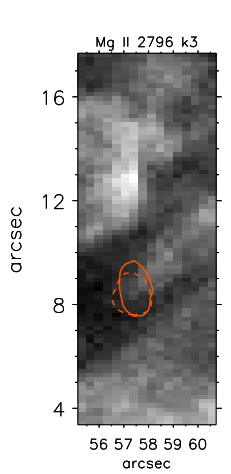

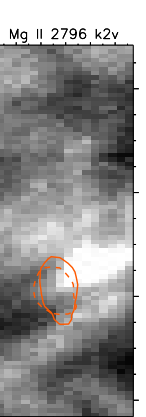

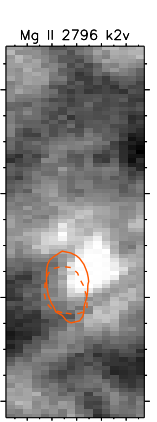

$\operatorname{arcsec}$

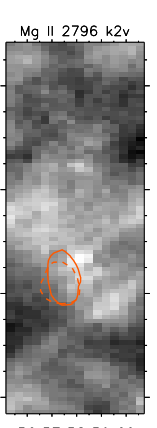

5657585960
08:09:05

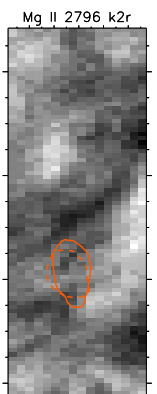

$\operatorname{arcsec}$

08:10:08

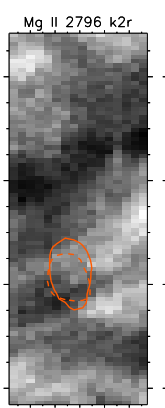

$\operatorname{arcsec}$

$08: 12: 13$

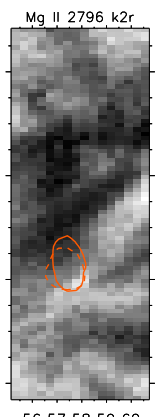

5657585960

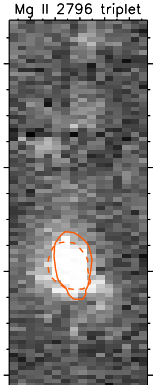

$\operatorname{arcsec}$

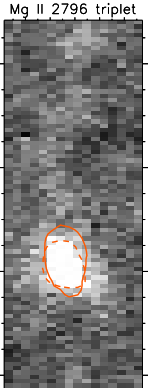

$\operatorname{arcsec}$

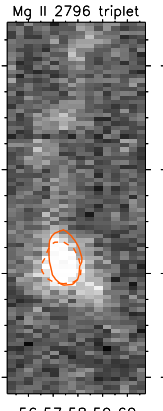

5657585960

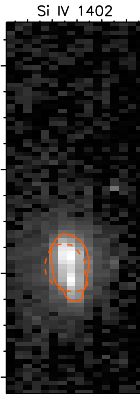

$\operatorname{arcsec}$

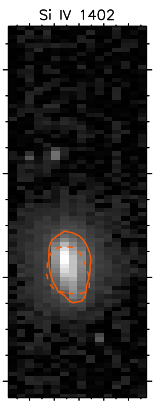

$\operatorname{arcsec}$

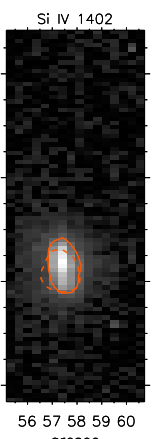

Fig. 6. Raster intensity maps corresponding to the nominal positions of the Mg II k3, k2v, k2r spectral features, the Mg II triplet, and the Si IV $140.3 \mathrm{~nm}$ line, for three time steps around the maximum intensity peak of the UV burst. The solid orange contours delimit the UV burst as seen in the Si IV intensity, while the dashed orange contours mark the boundaries of the burst as seen in the $\mathrm{Mg}$ II triplet line feature. We have applied a gamma adjustment to the Si IV color table.

behavior. The situation changes in the $\mathrm{k} 2 \mathrm{v}$ emission peak: at this wavelength we partially see a broader bright feature located in the same region as the Si IV burst, but only for the first two rasters at 08:09:05 and 08:10:08 UT. This brightening is not visible in the last raster (only guessed) presumably because the aforementioned loops cover the brightening. Finally, the $\mathrm{Mg}$ II triplet $279.6 \mathrm{~nm}$ line shows the full extent of the UV burst, which as stated above is wider than what is seen in Si IV maps. In fact, the UV burst in the Mg II triplet has a width of approximately $2^{\prime \prime}$ (from $x=56.5$ to $58.5^{\prime \prime}$ ), while in Si IV is barely $1^{\prime \prime}$ wide (from $57^{\prime \prime}$ to $58^{\prime \prime}$ ). At the same time the associated EB shows, in $\mathrm{H} \alpha$, an elongated shape during some time steps alternating to a more roundish shape at other time steps. Its size is smaller than the UV burst (around 1-1.5" of maximum length and $0.5-1^{\prime \prime}$ in width). This shape and size difference with rising temperature is explained by the expansion of the magnetic field with height; a few hundred kilometers above the photosphere where EBs are formed the magnetic field is confined by plasma motions in the high plasma- $\beta$ environment, but at greater heights the magnetic field expands and the current sheet that generates the EB and UV burst attains a wedge-like shape wider at the top than at the bottom (see Paper I) allowing for more extended brightenings.

Finally, Fig. 7 presents Doppler shift maps of the Mg II k3, $\mathrm{k} 2 \mathrm{v}$ and $\mathrm{k} 2 \mathrm{r}$ line features for the same three time steps shown in Figs. 4 and 6. These maps measure the observed departure of the particular spectral feature with respect to their laboratory rest wavelength. In order to determine the positions of the maximums and minimums along the $\mathrm{Mg}$ II $\mathrm{k}$ spectral line we have first smoothed the profiles both in the $y$-direction and in the $\lambda$-direction to avoid fluctuations that make it difficult for the fitting routine to find the proper $\mathrm{k} 3, \mathrm{k} 2 \mathrm{v}$, and $\mathrm{k} 2 \mathrm{r}$ spectral features. While most of the maps present smooth variations of the velocity values across the FOV, some pixels above and below the UV burst show extreme values with abrupt changes from positive to negative values. Those pixels correspond to $\mathrm{Mg}$ II profiles in which the spectral features cannot be identified due to their atypical shape. The solid black contours delimit the UV burst as seen in the Si IV intensity, while the dashed black contours mark the boundaries of the burst as seen in the $\mathrm{Mg}$ II triplet line feature.

Unlike the Si IV $140.3 \mathrm{~nm}$ Doppler shifts shown in Fig. 4, the Mg II Doppler shifts do not have differentiated zones within the UV brightening domain. In this case the Mg II line profiles (like the ones shown in Fig. 3) have much broader wings than the profiles at rest. The $\mathrm{k} 3$ minimum presents almost no Dopplershift, the $\mathrm{k} 2 \mathrm{v}$ maximum is blueshifted with respect to the profiles at rest (with peak values of $-40 \mathrm{~km} \mathrm{~s}^{-1}$ ), and the $\mathrm{k} 2 \mathrm{r}$ maximum is redshifted with respect to the profiles at rest (with maximum values of up to $40 \mathrm{~km} \mathrm{~s}^{-1}$ ) for all time steps. The shifts in $\mathrm{k} 2 \mathrm{v}$ and $\mathrm{k} 2 \mathrm{r}$ are not the result of monolithic mass motions, but are due to other reasons. The entire region around the EB/UV burst phenomena is characterized by broad wings in the $\mathrm{Mg}$ II k2 peaks, both on the blue side of the $\mathrm{k} 2 \mathrm{v}$ peak and on the red side of the $k 2 r$ peak. Spectral inversions of this line using the IRIS $^{2}$ package $^{1}$ (Sainz Dalda et al. 2019; de la Cruz Rodríguez et al. 2016) attribute the broad asymmetric $\mathrm{Mg}$ II h \& k peaks most likely to the following factors in the region surrounding the EB/UV burst: a highly turbulent plasma, with turbulent velocities $v_{\text {turb }}$ of $10-15 \mathrm{~km} \mathrm{~s}^{-1}$, covering an area that is larger than the UV burst seen in the other plasma variables like temperature $T$, line-of-sight velocity $v_{\text {los }}$, and electron density $n_{\mathrm{e}}$. The maximum of $v_{\text {turb }}$ is reached in the lower chromosphere, where $\log \left(\tau_{500}\right) \simeq-3$ (where the photosphere is defined by the height at which the optical depth $\tau=1$ in the continuum at $500 \mathrm{~nm}$, hence the notation $\tau_{500}$ ). A region of smaller horizontal extent than that covered by $v_{\text {turb }}$, outlining the high intensities seen in Fig. 6, shows electron densities of $n_{\mathrm{e}} \simeq 10^{13} \mathrm{~cm}^{-3}$, which is an order of magnitude greater than the ambient densities. Filling the same extent, the inverted temperature rises to $7000 \mathrm{~K}$ at $\log \left(\tau_{500}\right) \simeq-3$, some $2000 \mathrm{~K}$ hotter than ambient and remaining roughly constant up to $\log \left(\tau_{500}\right) \simeq-6$. The velocities inferred from the inversions in the same vicinity are found to be upflowing and of order $-10 \mathrm{~km} \mathrm{~s}^{-1}$ to $-20 \mathrm{~km} \mathrm{~s}^{-1}$. This pattern fills the lower to middle chromosphere $\left(-2>\log \left(\tau_{500}\right)>-4\right)$. Overlying this turbulent, dense and relatively hot plasma, IRIS ${ }^{2}$ finds that the turbulent velocities and densities decrease while the upflow velocity gradually decreases towards zero. In the upper chromospheric emission $\left(\log \left(\tau_{500}\right)<-6\right)$ one sees downflowing plasma with a $u_{\text {los }} \simeq 10 \mathrm{~km} \mathrm{~s}^{-1}$ redshift that outlines the diagonal fibrils

http://iris.lmsal.com/iris2/ 

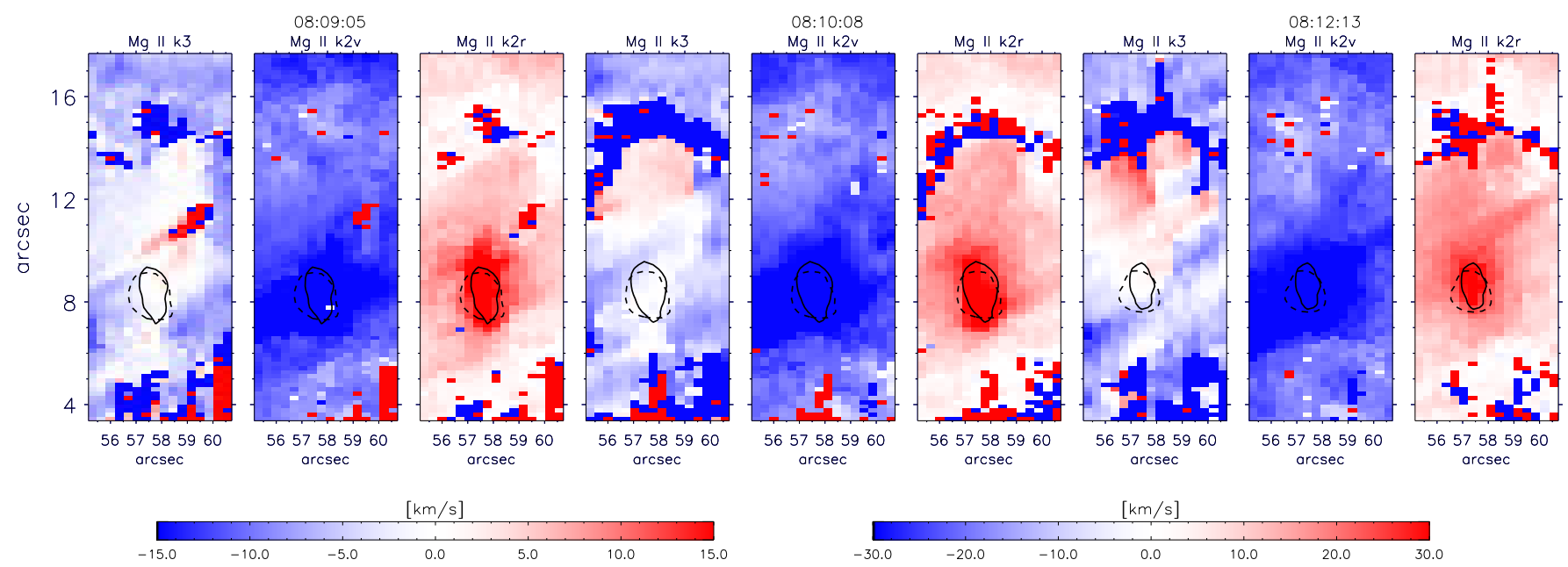

Fig. 7. Maps of the Mg II k Doppler shifts for three different time steps. For each time there are three panels representing the shifts with respect to the rest wavelength of the $\mathrm{k} 3, \mathrm{k} 2 \mathrm{v}$, and $\mathrm{k} 2 \mathrm{r}$ features of the $\mathrm{Mg}$ II $\mathrm{k}$ line. The color table on the left relates to the $\mathrm{k} 3$ spectral feature while the color table on the right refers to the $\mathrm{k} 2 \mathrm{v}$ and $\mathrm{k} 2 \mathrm{r}$ features. The solid black contours delimit the UV burst as seen in the Si IV intensity, while the dashed black contours mark the boundaries of the burst as seen in the $\mathrm{Mg}$ II triplet line feature.

visible in $\mathrm{H} \alpha$ and the $\mathrm{k} 3$ and $\mathrm{h} 3$ cores. One possible interpretation of the Doppler shifts found in the $\mathrm{k} 2 \mathrm{v}$ and $\mathrm{k} 2 \mathrm{r}$ peaks is then that the reconnection associated with the EB and UV burst generates turbulent wave motions that propagate into the surrounding medium in a wider area than actually covered by the hot, dense plasma, while at the same time accelerating the plasma upwards and heating it in a narrower region around the current sheet. The inverted atmospheres found for the broad Mg II k2 and h2 peaks in the vicinity of the EB and UV burst yield very similar results for all the rasters we have examined.

The excellent temporal cadence of the IRIS observations allows us to discern the temporal evolution of the UV burst properties. During the $15 \mathrm{~min}$ that we observe the UV burst in our observations, 90 IRIS SJIs (both in $140 \mathrm{~nm}$ and in $279.6 \mathrm{~nm}$ ) capture its evolution at a $10 \mathrm{~s}$ cadence. As mentioned above, the SJIs show a UV brightening with a predominantly circular shape that does not change significantly during its lifetime. In the spectral rasters the burst is smaller. The burst moves slightly towards disk center during the observations: a total of $-0.7^{\prime \prime}$ in the $x$-direction and $-1.4^{\prime \prime}$ in the $y$-direction. The Si IV velocities reach peak values immediately before the maximum intensity at 08:10 UT and then decrease again, a behavior also seen in the $\mathrm{Mg}$ II velocities.

\subsection{Coexistence of an EB and a UV burst: September 5, 2016}

This case of transient brightenings on September 5, 2016, also showcases the coexistence of an EB and a UV burst both spatially and temporally. The coordinates of these transient events are as follows: the initial position of the $\mathrm{EB}$ was $(x, y)=$ $(-162.5,39.5)^{\prime \prime}$ starting at 09:26:04 UT and the initial position of the UV burst was $(x, y)=(-162.5,39.8)^{\prime \prime}$ at 09:26:36 UT. Figure 8 presents the mosaic of SST, IRIS, and SDO observations that shows the context in which these brightenings were produced. The SST images in Fig. 8 were taken at 09:29:50 UT, the IRIS SJI $140 \mathrm{~nm}$ at 09:29:45 UT, and the SJI $279.6 \mathrm{~nm}$ at 09:29:48 UT. Finally, the SDO imagery was taken at 09:31:59 UT. A flux emergence region is situated in the center of the FOV, east of the penumbra seen in the right side of the panels and northwest of the small penumbrae observed on the left side of the panels. As in Fig. 1, the EB is marked with a red contour and the UV burst with a yellow contour. The location where these brightenings occur coincides with fields of opposite polarity colliding with each other as can be seen in the Stokes V maps of Ca II $854.2 \mathrm{~nm}$ or the HMI magnetograms of Fig. 8. An extended patch of negative polarity interacts with a very small patch of positive polarity.

In the SST images, the EB reaches a maximum size of around $1^{\prime \prime} \times 1.5^{\prime \prime}$ with a highly variable and irregular shape throughout its lifetime. The UV burst, which in the SJIs always lies on top of the EB, maintains a roundish shape throughout its lifetime and peaks at a diameter of $2.5^{\prime \prime}$. The burst is observed both in the NUV SJI at $279.6 \mathrm{~nm}$ as well as in the FUV SJI at $140 \mathrm{~nm}$.

As in the previous case (and in all cases where a UV burst is present, see Table 2) the $\mathrm{H} \alpha$ wing images at $-0.1 \mathrm{~nm}$ show a surge shooting diagonally from the EB in the northeast direction at 09:27:09 UT and lasting for $6 \mathrm{~min}$. The maximum length of the surge is $9^{\prime \prime}$ in the $\mathrm{H} \alpha-0.1 \mathrm{~nm}$ images and $12^{\prime \prime}$ in the $\mathrm{H} \alpha$ core images. The temporal evolution of the surge can be followed in the $\mathrm{H} \alpha$ and $\mathrm{Ca}$ II $854.2 \mathrm{~nm}$ movies available online.

The response of the AIA transition region and coronal channels is very similar to that of the case introduced in Sect. 3.1, that is, no signature associated to these reconnection events is observed at those hotter EUV wavelengths. The surge on the other hand leaves a visible imprint in the form of a darkening with the same shape. Again the cold dense material of the surge absorbs light and does not allow the radiation escaping from the reconnection site to reach coronal heights. However, at much lower chromospheric temperatures, the AIA $170 \mathrm{~nm}$ channel displays a small brightening at the co-spatial position of the EB and the UV burst.

Figure 9 illustrates the temporal evolution of the EB and UV brightening intensities during their coexistence. As in Fig. 2, the upper panel displays the intensities of the EB in $\mathrm{H} \alpha$ (wing and line core) and in Ca II $854.2 \mathrm{~nm}$ (wing and line core). The middle panel shows the intensity derived from the IRIS SJIs $279.6 \mathrm{~nm}$ while the lower panel presents the intensity obtained from the SJI $140 \mathrm{~nm}$ images (see details on the identification and tracking of events and how light curves are calculated in Sects. 2.2 and 2.3). We note that in this case the IRIS observations are affected by SAA events at the end of the observing period and those can be 

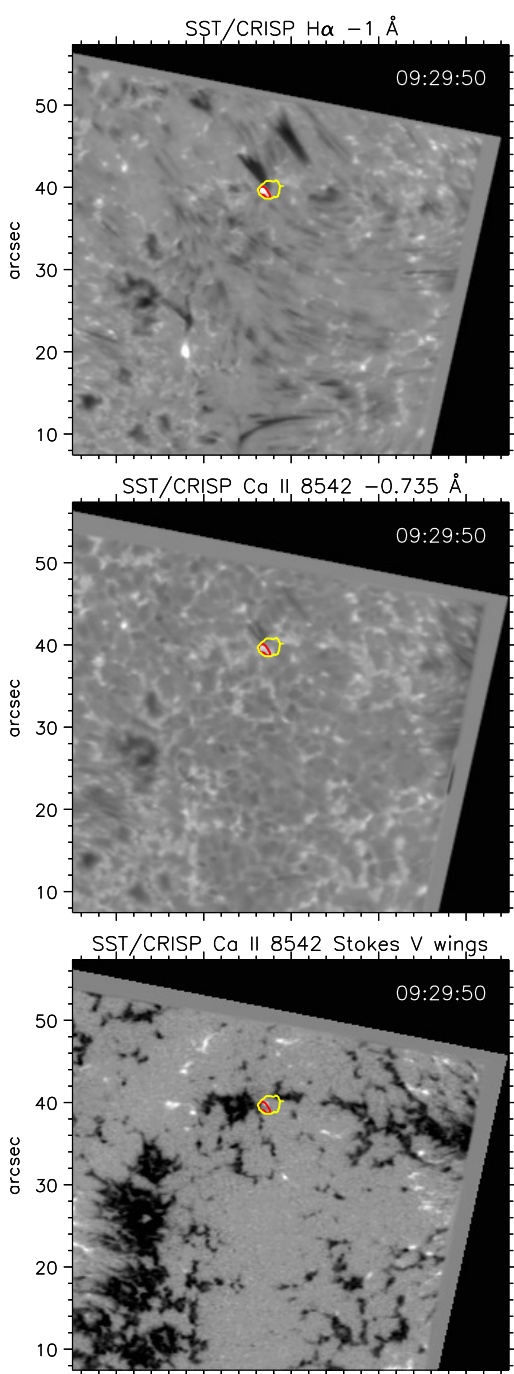

SDO/AIA $304 \AA$

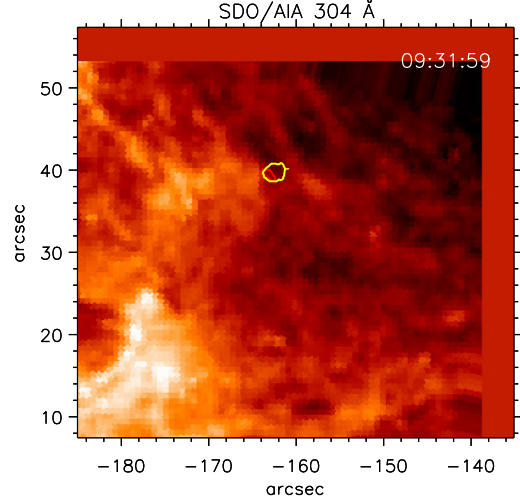

SST/CRISP, $H \alpha+0 . \AA$

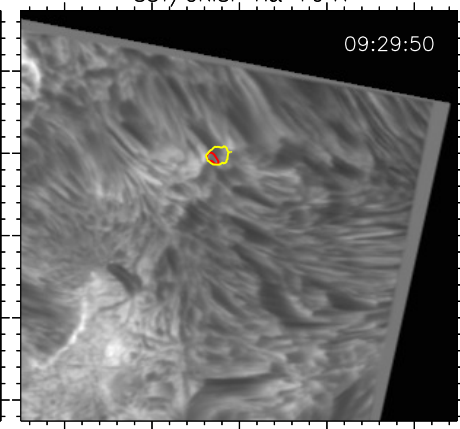

SST/ÇRISP Ca $\| 1 / 8542$ +0 $\AA$

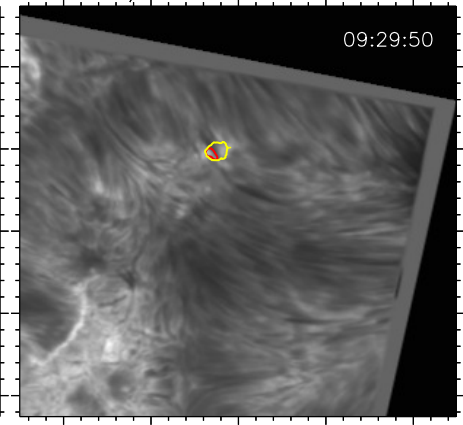

SDQ/HMI mognetogram

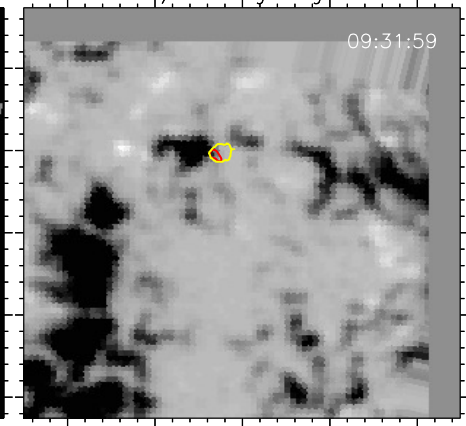

SDO/AIA $171 \AA$

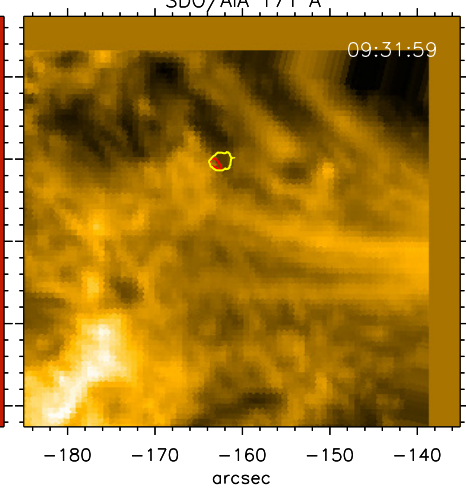

IRIS/SJI $2796 \AA$

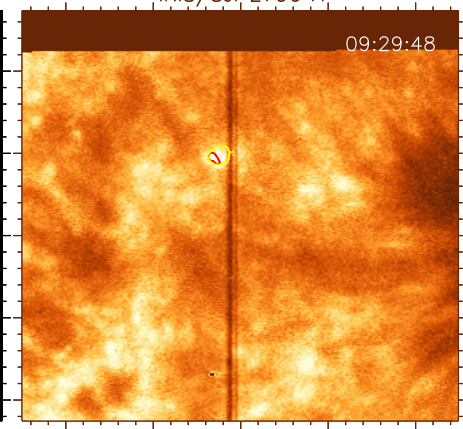

IRIS/SJI $1400 \AA$

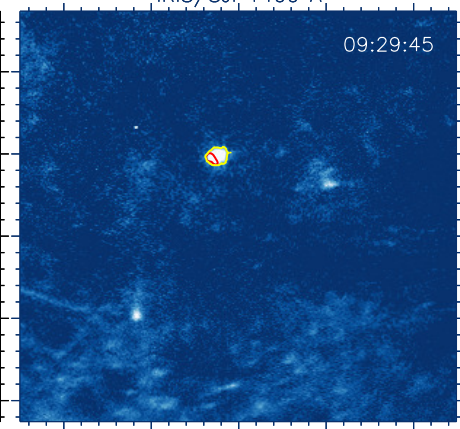

SDO/AIA $1700 \AA$

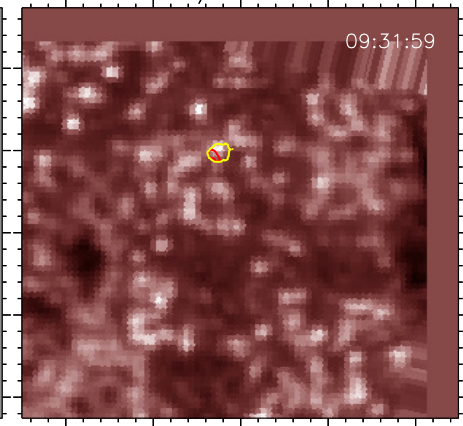

SDO/AIA $193 \AA$

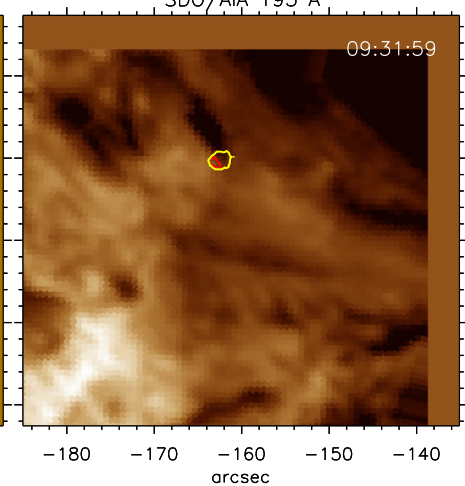

Fig. 8. Same as Fig. 1 but for September 5, 2016. At this particular time both an EB and a UV burst co-existed co-temporally and co-spatially in the FOV.

seen as outlier peaks in both UV intensities. The beginning of the SAA period is marked by red dashed vertical lines in Fig. 9.

The EB is present during almost the whole observation period, but it shows a peak in intensity between 09:26:04 and 09:33:35 UT (with a maximum value at 09:28:13 UT). At the end of the observing period the EB is still visible on the solar disk. Almost simultaneously (only $30 \mathrm{~s}$ later) the burst in NUV and FUV lights up at 09:26:36 UT lasting for $7.5 \mathrm{~min}$. At the end of the observation the UV burst is still slightly visible in the SJI $140 \mathrm{~nm}$. The maximum intensity is observed between 09:29:14 and 09:29:35 UT in SiIV (lower panel) and at 09:29:35 UT in
Mg II (middle panel), that is, $80 \mathrm{~s}$ after the maximum in $\mathrm{H} \alpha$ wing and Ca II $854.2 \mathrm{~nm}$ wing. However, we note that the line core of $\mathrm{H} \alpha$ peaks at the same time as the UV intensities.

To complete this case, we present in Fig. 10 the spectral information gathered from the SST and IRIS observed spectra. As in Fig. 3, the left panel shows spectral profiles for $\mathrm{H} \alpha$ (top panel), $\mathrm{Mg}$ II $\mathrm{k}$, and $\mathrm{h}$ lines at $279.6 \mathrm{~nm}$ (middle panel), and the Si IV lines at 139.4 and $140.3 \mathrm{~nm}$ respectively (bottom panels), for four selected time steps. These times represent approximately: the onset of the EB (09:25:32 UT, black line), the onset of the UV burst (09:26:36 UT, blue line), maximum brightness of 

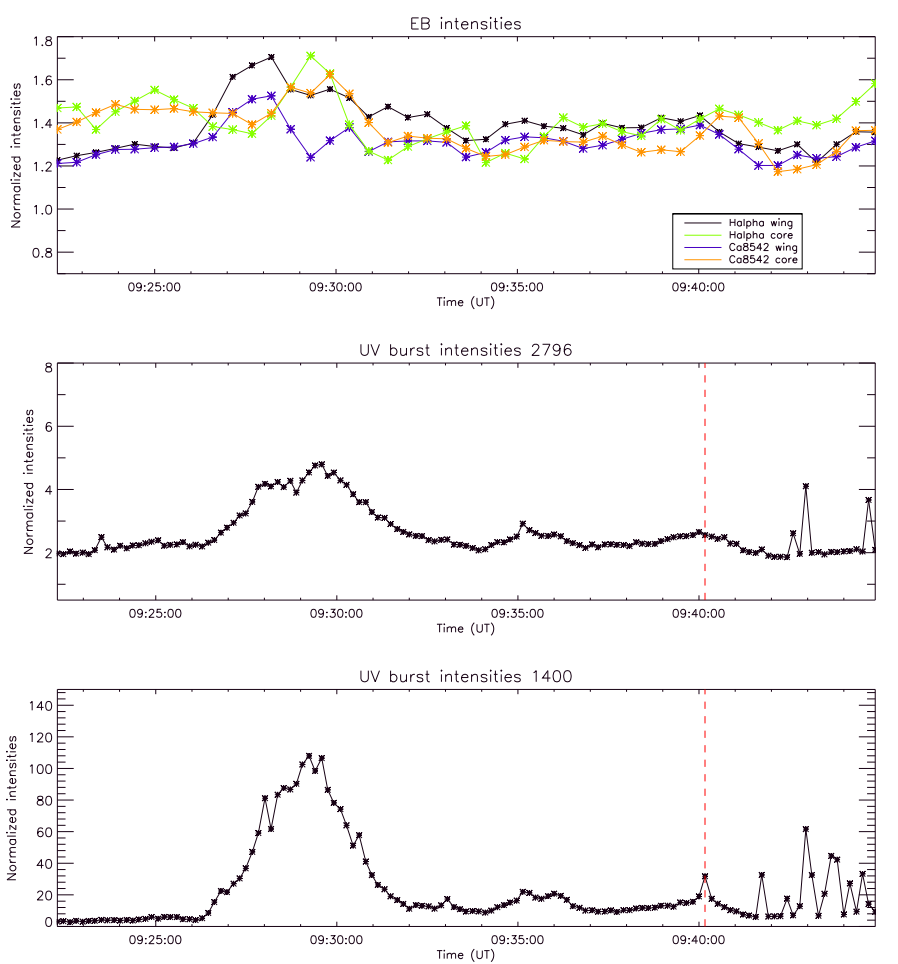

Fig. 9. Same as Fig. 2 but for September 5, 2016. The red vertical dashed line marks the start of the passage of the IRIS satellite through the South Atlantic Anomaly where the detectors encounter increased noise levels due to cosmic ray hits.

the EB (09:28:06 UT, green line), and finally maximum brightness of the UV burst (09:29:29 UT, red line). The right side of the figure is a time-sliced spectrum that displays the temporal evolution of three spectral lines: SST $\mathrm{H} \alpha$, IRIS Mg II k and triplet lines, and IRIS Si IV $139.4 \mathrm{~nm}$. The red arrow in the $\mathrm{H} \alpha$ spectra highlights the onset of the EB, and the red arrows in the IRIS spectra mark the onset of the UV burst.

The upper left panel shows that the $\mathrm{H} \alpha$ spectra are quite similar at all times, with the exception of 09:28:06 UT (green curve, moment of maximum brightness of the EB), when the wings of $\mathrm{H} \alpha$ exceed $150 \%$ of the intensity of a quiet Sun profile; the left wing reaches $170 \%$ of that intensity. It is indeed that same time step (09:28:06 UT) which presents the highest emission in the $\mathrm{Mg}$ II triplet in the central left panel of Fig. 10. Both the Mg II spectra taken at 09:28:06 and 09:29:29 UT show a very slight absorption due to Mn I superimposed on the blue wing of $\mathrm{Mg}$ II k profiles. This absorption is not visible in the other two time steps (which coincide with the onset of the EB and UV burst) as their profiles are not broad enough to present absorption lines. Inspection of the Si IV spectra in the lower left panels reveals that only these same time steps (09:28:06 and 09:29:29 UT) have a significant amplitude in the FUV. Only the 09:29:29 UT profile is wide enough to present Ni II absorption lines at $-93 \mathrm{~km} \mathrm{~s}^{-1}$ superimposed in the Si IV $139.4 \mathrm{~nm}$ profiles.

This scenario suggests that in the beginning of the observation the atmosphere is not hot enough. It is only around 09:27 UT and beyond that the lower chromosphere is hot enough to produce emission in the triplet of $\mathrm{Mg}$ II and later on heats even more to produce a significant intensity amplitude in the FUV $\mathrm{Si}$ IV profiles. This sequential behavior is also seen in the timesliced spectrum (right side of Fig. 10): first the EB lights up maintaining its bright wings for about 2.5 minutes (the right wing is bright for a longer time), then the NUV lights up $30 \mathrm{~s}$ after the EB, and subsequently the FUV starts heating up gradually reaching a significant intensity from 09:28 UT onwards.

\subsection{Ellerman bomb not associated with a UV burst: September 5, 2016}

The event introduced in this section presents a case of an EB not associated to any UV brightening, either temporally or spatially. This EB was present on the solar disk on September 5, 2016. Its initial position was $(x, y)=(-158.0,3.5)^{\prime \prime}$ at 08:16:36 UT. This is the only case of this study that does not have a surge associated with it. The EB is the site of interaction between magnetic fields of opposite polarities. In particular an extended and still negative polarity patch intermittently interacts with tiny positive polarity patches that constantly emerge at the edge of the negative patch.

The EB of this case reaches a maximum size of $1^{\prime \prime} \times 1^{\prime \prime}$, varying along its life time. During the $16.5 \mathrm{~min}$ of life its size peaks at 08:19 and 08:22 UT. Its shape is irregular and also changes with time. No signature of this event is observed in the IRIS SJIs or spectra.

Figure 11 presents the temporal evolution of the intensity of this EB. The layout of the figure is the same as in Figs. 2 and 9. Intensity in the $\mathrm{H} \alpha$ and $\mathrm{Ca}$ II $854.2 \mathrm{~nm}$ wing narrowband filtergrams is very similar and does not reach such high values as the previous case. However, the $\mathrm{H} \alpha$ line core (green curve) shows a dip in intensity around the time of maximum brightness of the EB (as measured by the wings at 08:23 UT) which is not observed in the center of the Ca II 854.2 nm line. From 08:22:20 to 08:27:24 UT an unrelated surge (whose feet lie around 4" northwest from our event) covers the EB in its entirety causing a decrease in the $\mathrm{H} \alpha$ core intensity. The presence of that surge and its evolution can be seen in the online movies. The EB starts at around 08:17 and ends at 08:26 UT thus lasting for $9 \mathrm{~min}$. The UV intensity obtained from the IRIS SJIs (middle and lower panels) shows no trace of the existence of any brightening.

Figure 12 gives the spectral information on this event obtained from SST and IRIS at three different time steps: beginning of the EB (black curve, 08:16:42 UT), approximate moment of maximum brightness of the EB (blue curve, 08:22:16 UT), and decay of the EB (red curve, 08:27:29 UT). The outlay of the figure is the same as in Figs. 3 and 10. In the period of peak brightness the wings of $\mathrm{H} \alpha$ climb up to $160 \%$ compared to the intensity of a quiet Sun profile. Both wings appear very symmetric. The profiles from the beginning and end of the life of the EB (black and red curves) are only $110 \%$ brighter than that of a quiet Sun pixel. Both the NUV and FUV profiles in the middle and lower panels show no significant brightening. Only a marginal intensity increase is observed in the $\mathrm{Mg}$ II triplet at a particular time step. The time-sliced spectrum in the right side of Fig. 12 shows only the typical spectral evolution of an EB whose peak intensity lasts for about three minutes. No apparent heating of the upper atmospheric layers can be measured in this case.

\subsection{Ultraviolet burst not associated with an EB: September 5, 2016}

The last case we show in detail is an event where only a UV burst was observed and no EB was found co-temporally or co-spatially. On September 5, 2016, a UV brightening was observed in the solar disk from 08:53 until 09:19 UT. The brightening was indeed already visible at the start of the data set. Its initial position was $(x, y)=(-160.7,37.1)^{\prime \prime}$ at 08:53:00 UT. This event is introduced in Fig. 13 which sets the scenario surrounding this event. The FOV is the same as in Sect. 3.3 but at an 

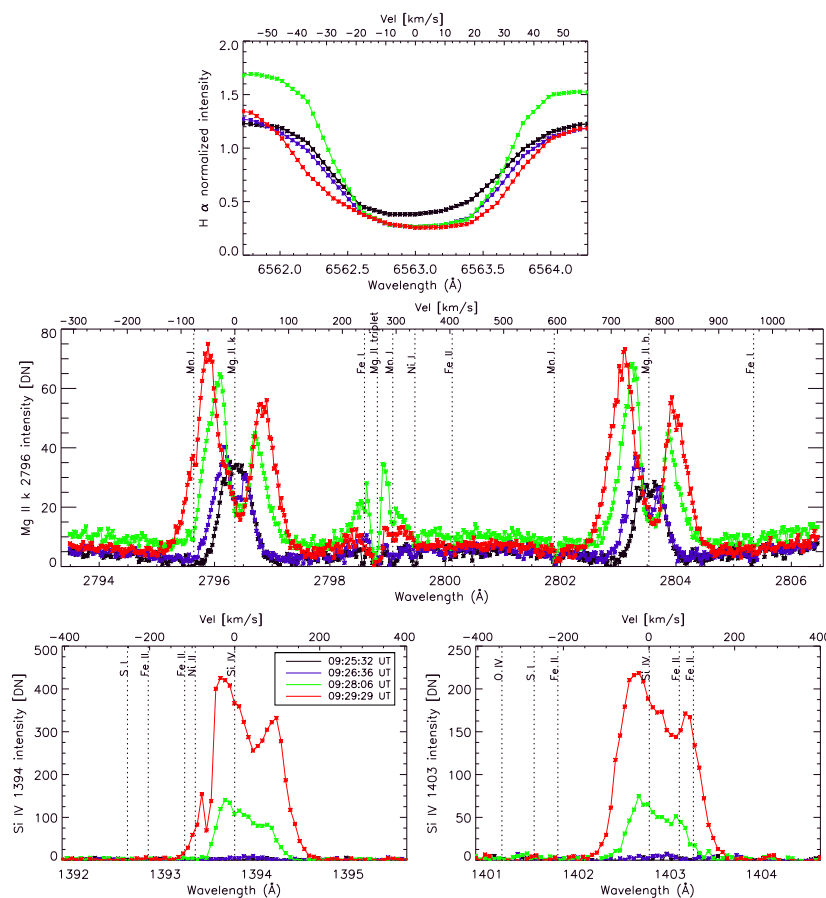
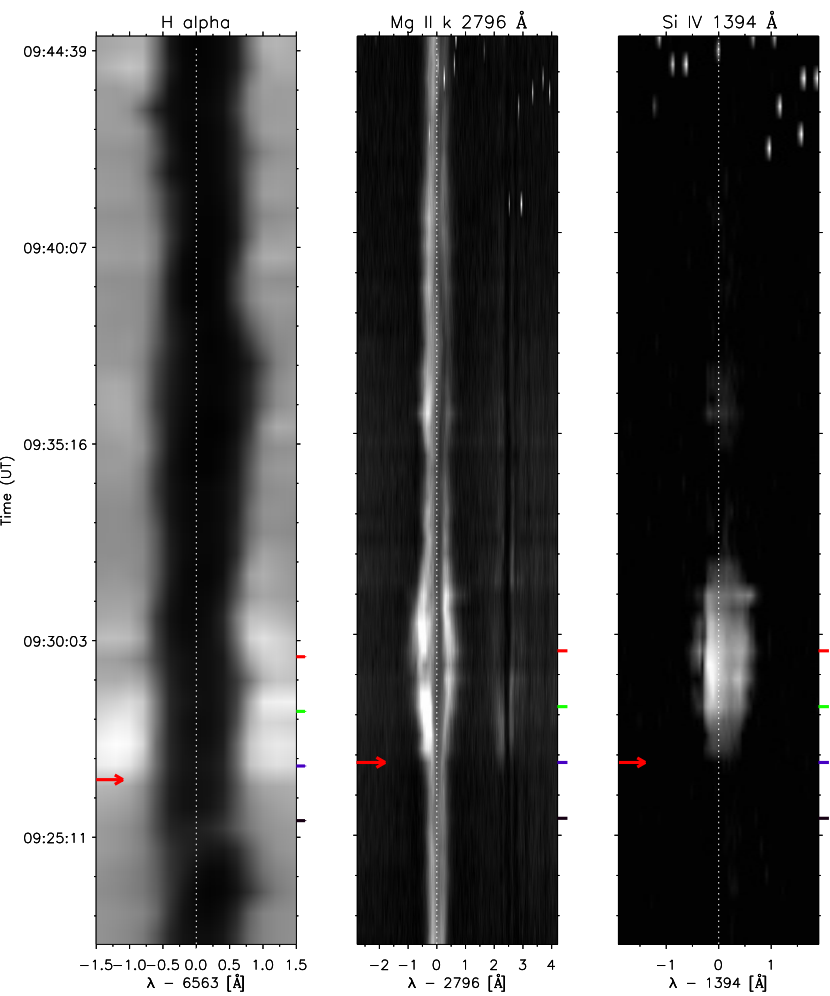

Fig. 10. Same as Fig. 3 but for September 5, 2016. The colored marks in the time-sliced spectra pinpoint to the same times that have been highlighted in the left panel. We have applied a gamma adjustment to the Si IV color table.
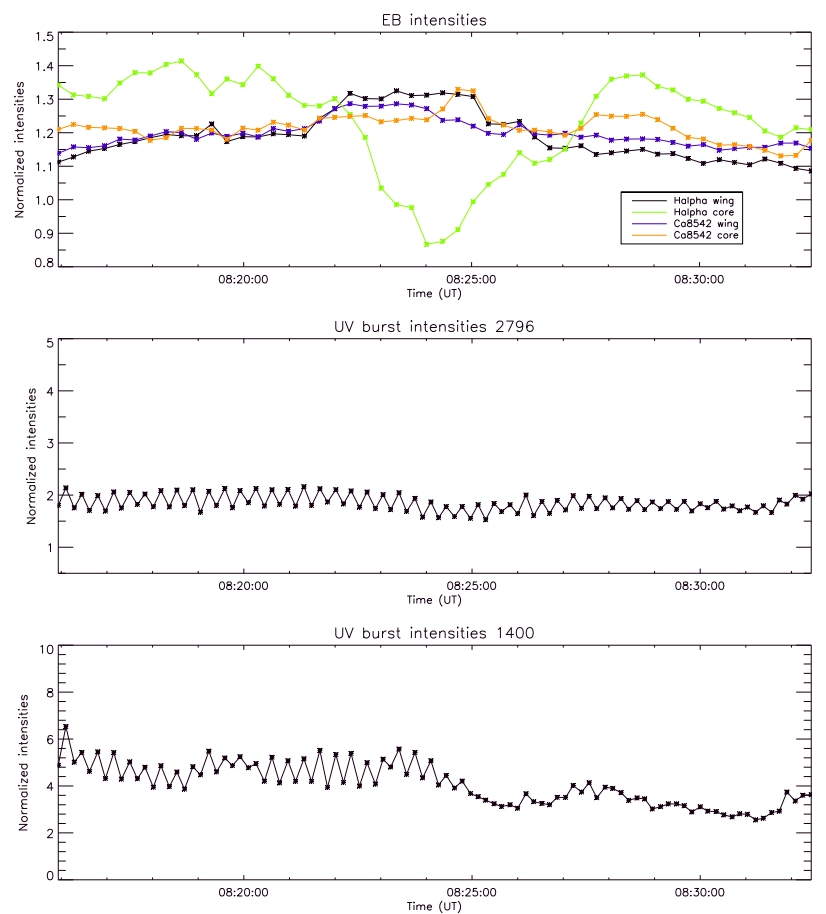

Fig. 11. Same as Fig. 2 but for September 5, 2016. In this case only an EB was present and no UV brightening could be associated with it.

earlier time, that is, an area of active flux emergence between two sunspots. The Stokes $V$ movies in the wings of Ca II $854.2 \mathrm{~nm}$ show several small positive-polarity patches traveling eastwards away from the sunspot in the upper right of the images and interact with the more extended negative-polarity patch which lies at around $(x, y)=(-166,40)^{\prime \prime}$. In Fig. 13 the displayed SST and
SDO observations are taken at 09:05:40 UT, while the IRIS panels are taken at 09:05:47 and 09:05:49 UT for the Si IV and Mg II SJIs respectively. In this figure the UV burst is highlighted with a yellow contour that has been superimposed on all panels of the figure. The UV brightening shows a peak size of $3^{\prime \prime} \times 2^{\prime \prime}$ at 08:57:36 and at 09:03:31 UT. The brightening has a very bursty behavior (see details in Sect. 3.2), reaching several maximums of intensity and size as can be seen in the IRIS SJI movies, as well as in Fig. 14.

The $\mathrm{H} \alpha$ wing panel at $-0.1 \mathrm{~nm}$ shows a surge shooting out diagonally from the lower-left part of the burst, which remains for the whole data set. The surge is also visible in the core of the $\mathrm{H} \alpha$ line. Like in the previous cases, the surge is a very dynamical structure with a maximum length of 7" (reached at 09:09:25 UT) in the $\mathrm{H} \alpha-0.1 \mathrm{~nm}$ images.

The SDO/AIA coronal channels do not show any signature of heating, but a darkening in coincidence with the surge. The AIA $170 \mathrm{~nm}$ panel of Fig. 13 shows a brightening at the position of the UV patch.

Figure 14 shows the temporal evolution of the intensity at several wavelengths for the duration of the UV burst. Outlay is similar to that of Figs. 2, 9 and 11. The wings of $\mathrm{H} \alpha$ and Ca II $854.2 \mathrm{~nm}$ (black and blue curves) present a flat behavior (typical for a case with no EB), while the centers of those lines show some peaks in coincidence with brightenings occurring at the feet of the surge. The NUV and FUV intensities (middle and lower panels) obtained from the IRIS SJIs evolve in unison and present a bursty behavior with up to four peaks during the span of the UV burst total lifetime (about $26 \mathrm{~min}$ ). During that time span, three bright periods of 6,7 , and 1 min in duration, respectively, can be distinguished in the Si IV intensity. The intensity variations at these wavelengths are, for this case, much lower when compared to the bursts of Sects. 3.1 and 3.3 when the UV bursts were associated with an EB. 

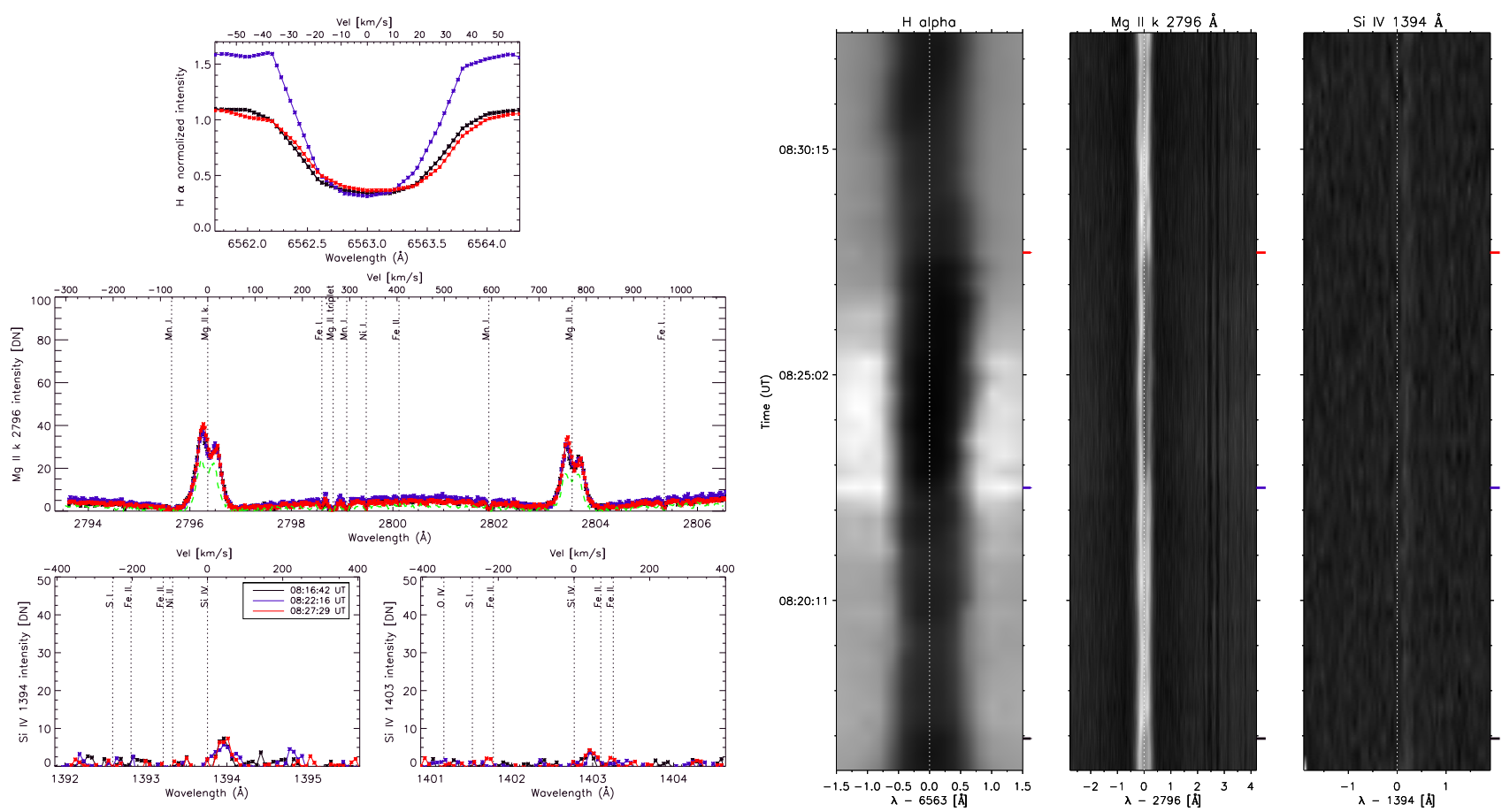

Fig. 12. Same as Fig. 3 but for September 5, 2016. No UV burst was found associated with this particular EB. The dashed green curve in the $\mathrm{Mg}$ II panel shows a quiet Sun profile for reference: barely any imprint from the EB is observed in the Mg II spectra. The colored marks in the time-sliced spectra pinpoint to the same times that have been highlighted in the left panel.

Finally, Fig. 15 shows the spectral profiles as well as their temporal evolution. The outlay is the same as in Figs. 3, 10, and 12. The left side of the figure shows the SST and IRIS spectra at six different times: beginning of the observations (black line, 08:53:01 UT), first peak of the UV burst (purple line, 08:57:11 UT), relaxation time between peaks (dark blue, 08:59:37 UT), second peak of UV burst (turquoise line, 09:04:08 UT), relaxation time between peaks (green line, 09:12:07 UT), and finally third peak of burst (red line, 09:18:01 UT). The $\mathrm{H} \alpha$ profiles (upper left panel) do not show any particular feature at any time other than a blueshift at 08:57:11 UT and a redshift at 08:59:37 UT (both Dopplershifts visible also in the time-sliced spectra). The UV spectra in the middle and lower left panels show the typical signature of a UV burst but at a much smaller scale than in the previously presented cases. No photospheric or chromospheric absorption lines are found superimposed to either the $\mathrm{Mg}$ II k and $\mathrm{h}$ profiles at $279.6 \mathrm{~nm}$ or the SiIV profiles at 139.4 and $140.3 \mathrm{~nm}$. Only the moments of peak intensity (purple, turquoise, and red curves) have a measurable amplitude in the Si IV profiles, while the periods of relaxation between peaks do not show a significant FUV profile.

The time-sliced spectra on the right side of Fig. 15 displays the temporal evolution of three spectral lines: SST $\mathrm{H} \alpha$, IRIS Mg II k and triplet lines, and IRIS Si IV $139.4 \mathrm{~nm}$. The red arrows mark the beginning of the different bright periods along the $26 \mathrm{~min}$ of lifetime. The $\mathrm{H} \alpha$ temporal evolution shows two periods of blueshifts lasting for about 1 and $8 \mathrm{~min}$, respectively. Those blueshifts roughly correspond to the periods of brightening in the UV wavelengths.

\subsection{Other cases in brief}

In addition to the four cases introduced in more detail in the previous sections, we also examined four more cases that we briefly report here. Two of these cases are events in which an EB and a
UV burst coexisted co-spatially and co-temporally, both of them occurring on September 3, 2016, at two different locations within the same FOV. In these particular cases the EB and the UV burst lit up almost simultaneously (within one min). On September 4 a case of a UV burst not associated to an EB was identified away from the IRIS slits. Finally our last case was on September 5 also showing a UV burst not accompanied by an EB. We believe that this UV brightening falls in the FAF (flaring arch filament) category (Vissers et al. 2015). All these UV brightenings share an intermittent (bursty) behavior when looking at the temporal evolution of their intensities and the online movies. In addition, all these four cases have surges associated to them. More details can be found in Table 2 .

Finally, we derived the velocities of our seven UV brightenings using the observed IRIS lines. The Si IV $140.3 \mathrm{~nm}$ velocities yield maximum values of $\pm 100 \mathrm{~km} \mathrm{~s}^{-1}$ for all cases, with averages around $\pm 50 \mathrm{~km} \mathrm{~s}^{-1}$. Regarding velocities derived from the IRIS Mg II lines, average values for the Mg II $\mathrm{k} 3$ minimum yield $\pm 5 \mathrm{~km} \mathrm{~s}^{-1}$. The velocities for the $\mathrm{k} 2 \mathrm{v}$ and $\mathrm{k} 2 \mathrm{r}$ maximums reach $\pm 30-40 \mathrm{~km} \mathrm{~s}^{-1}$ in all cases.

\subsection{Absence or presence of O Iv lines}

Peter et al. (2014) realized that the forbidden O IV 139.97 and $140.1 \mathrm{~nm}$ lines were absent from their identified UV bursts. Tian et al. (2016) noted that those lines were also absent or very weak in their UV brightenings associated with EBs, but present in the bursts not connected to EBs.

The existence of O IV lines and the ratio of Si IV $139.4 \mathrm{~nm}$ to O IV $140.1 \mathrm{~nm}$ provides a lower limit on the electron density in the source region of these lines (see, e.g. Peter et al. 2014). These authors used this method to conclude that the most likely reason for the absence of O IV lines in their observations of UV bursts is the high density in the bombs, reaching $3 \times 10^{14} \mathrm{~cm}^{-3}$ in the source region of $\mathrm{Mg}$ II, implying formation very low in 

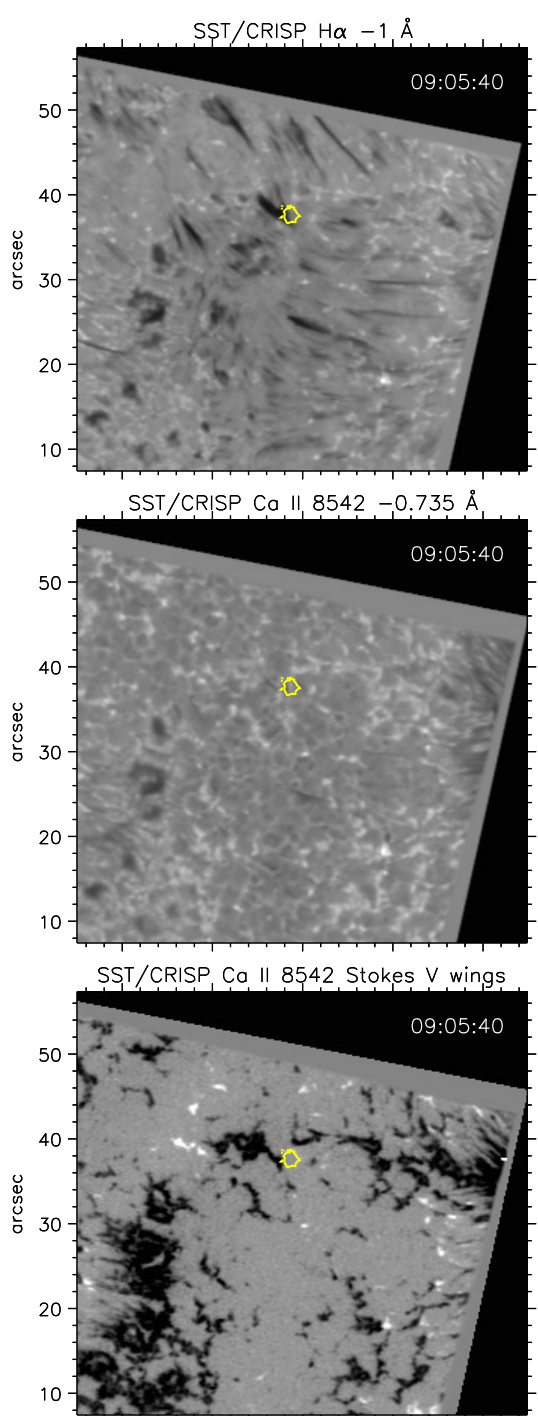

SDO/AIA $304 \AA$

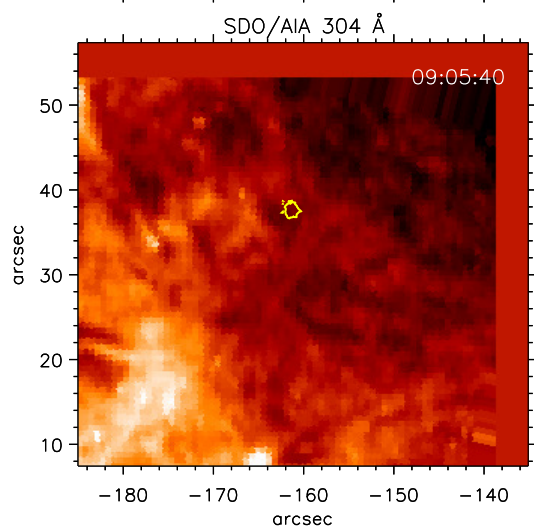

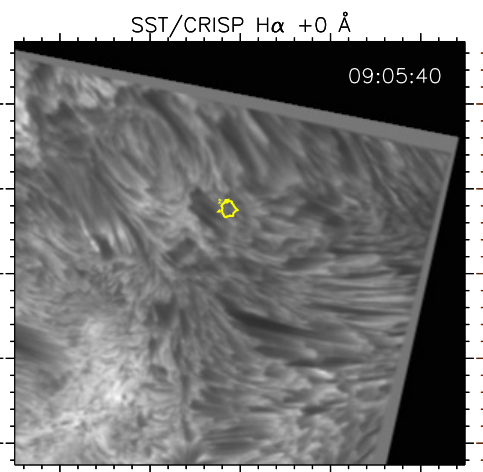

SST/CYRISP Ca $\| 8542+0 \AA$

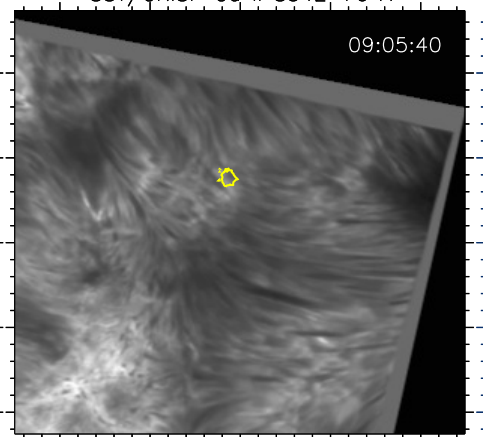

$\mathrm{SDO} / \mathrm{HMI}$ magnetogram

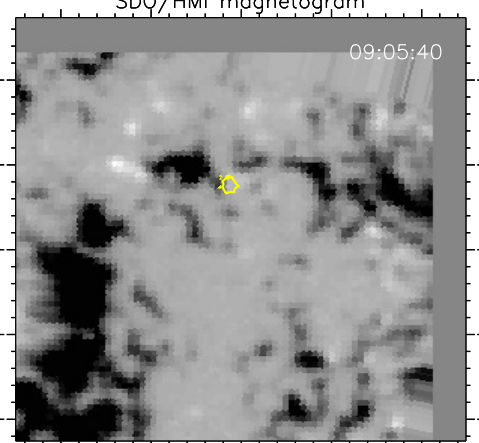

SDO/AIA $171 \AA$

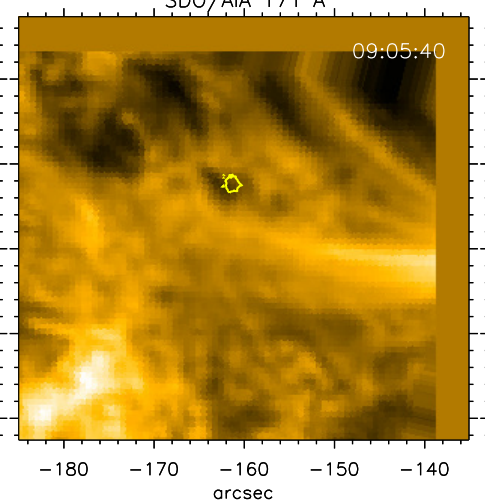

IRIS/SJI, $2796 \AA$

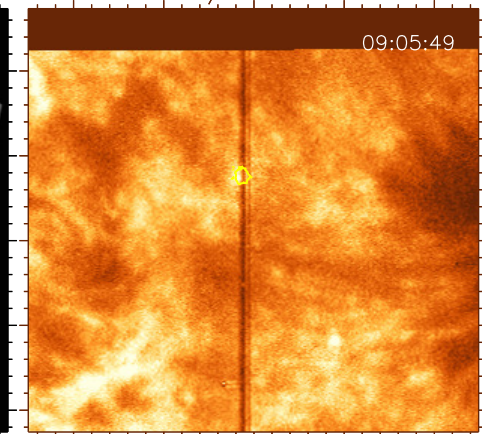

IRIS/SJI, $1400 \AA$

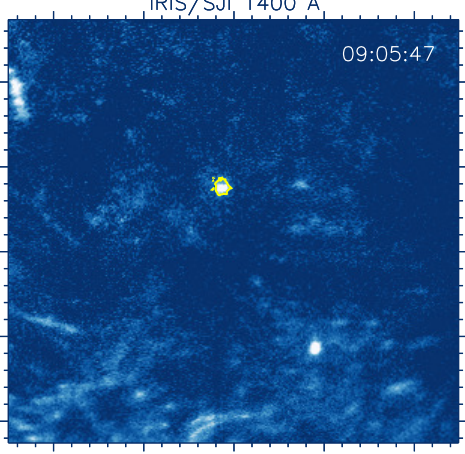

SDO/AIA $1700 \AA$

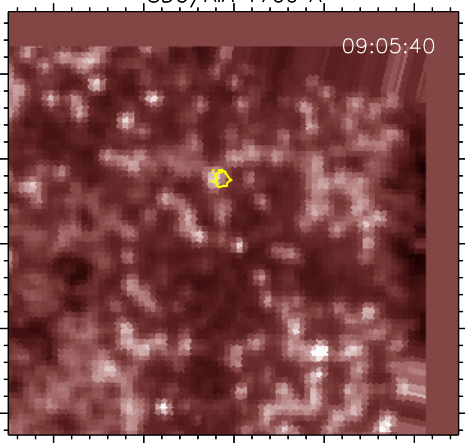

SDO/AIA $193 \AA$

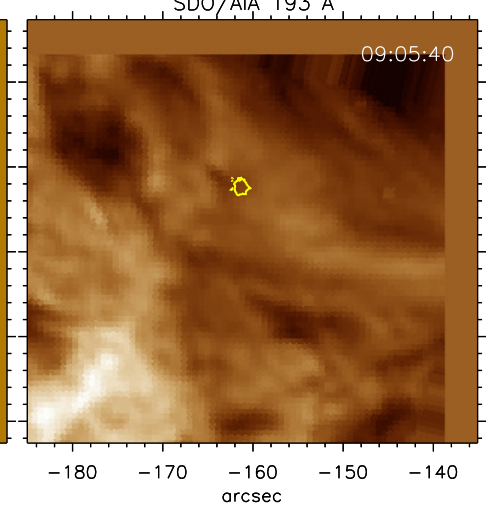

Fig. 13. Same as Fig. 1 but for September 5, 2016. In this FOV and at this time of the observation only a UV burst was present with no associated EB.

the solar atmosphere. Other justifications for this absence have been given: Olluri et al. (2013) attribute the absence of O IV lines to nonequilibrium ionization and Dudík et al. (2014) state that even at low densities such lines can be greatly suppressed in the presence of nonMaxwellian electron distributions.

Our IRIS observations do not allow us to draw conclusions on this particular subject because the exposure time of the IRIS spectral observations was only $0.5 \mathrm{~s}$, and those lines are faint and require longer exposure times (for reference, Tian et al. (2016) had a exposure time of $8 \mathrm{~s}$ ), resulting in profiles that are too noisy for accurate detection of any O IV signal above the noise level.

\section{Discussion and conclusions}

AR 12585, a region of abundant flux emergence which we observed in September 2016, has served the purpose of studying the temporal and spatial connection between transient dynamical 

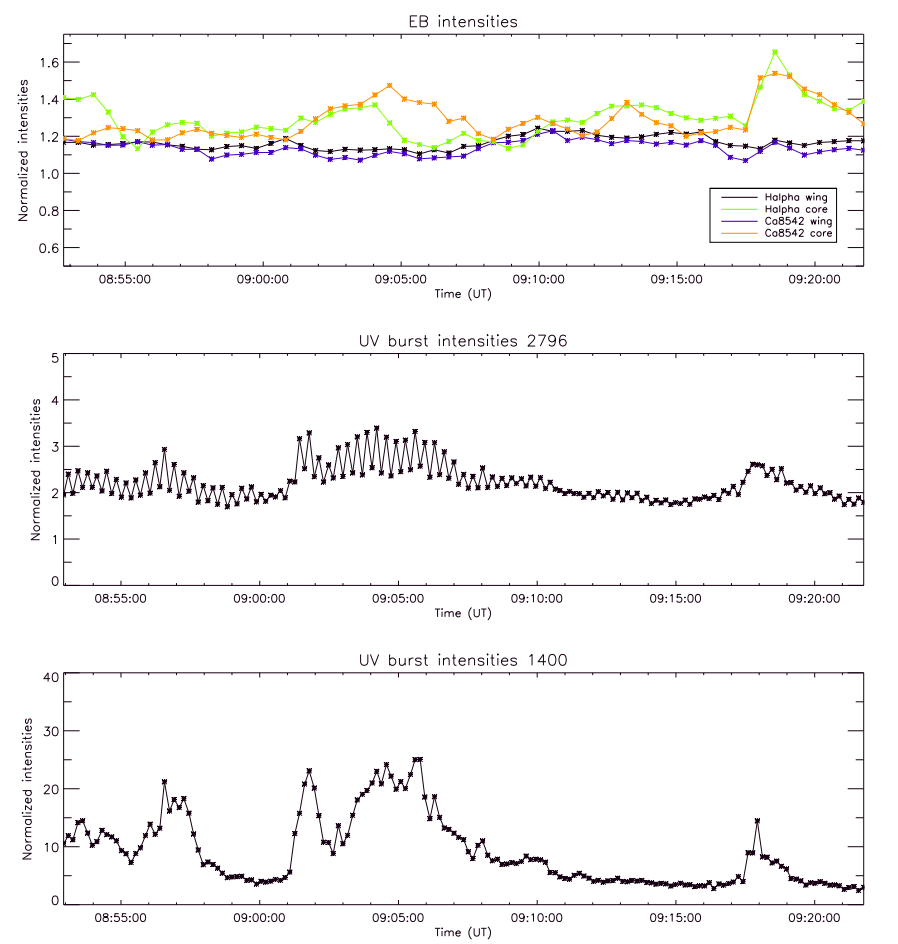

Fig. 14. Same as Fig. 2 but for September 5, 2016. At this time of the observation only a UV brightening was present with no EB associated with it.

phenomena resulting from magnetic reconnection, namely EBs and UV bursts. For this purpose we took, reduced, and analyzed high-cadence, high-resolution coordinated observations from the SST and IRIS. The eight cases studied allow us to establish a clear relationship between them, which we interpret in view of the results of the numerical Paper I.

We find that EBs are sometimes co-spatial and co-temporal with a UV burst, and sometimes not. The same is true for UV bursts, which are sometimes colocated and simultaneous with an EB, and sometimes not. In a given spectral image at a given time step we find that the occurrence of both events simultaneously and co-spatially is between 10 and $20 \%$. When these two phenomena occur together, they do so almost simultaneously (see e.g., the case described in Sect. 3.3) or with a delay of a few minutes (as in Sect. 3.1). Our observations (see Table 2) also show that whenever EBs and UV bursts coexist, the EB is always longer lived by several minutes, with the UV brightening appearing later (the delay being between only a few seconds and a few minutes). The intensity evolution of the UV burst presents a more rapid rise and fall - a more impulsive behavior - than its associated EB. The numerical simulations in Paper I point towards the same situation: the UV burst lights up and its temperature rises rapidly in the region between 500 and $3000 \mathrm{~km}$ and falls again after about two hundred seconds, while the EB exists for a longer period appearing before and disappearing after the UV burst. Both the light curves and the time-sliced spectra, which reveal the spectral history of our magnetic reconnection cases, make evident a sequential nature of heating events. These figures show that EB and UV burst intensities evolve in unison, but that there is a sequential behavior: first the $\mathrm{H} \alpha$ lights up, then the Mg II, and finally the Si IV. We thus observe a clear temporal and spatial relationship between these kind of events whenever they coexist. In view of the results from Paper I, this could be interpreted as a reconnection sequence happening from the bottom to the top of the long current sheet or, alternatively, as an indication that it takes more time to heat the atmosphere to transition region temperatures than to heat the denser plasma closer to the photosphere to emit in $\mathrm{H} \alpha$. Another result revealed by the light curves of the eight cases is that, in general, the intensity peaks of those EBs and UV bursts that are colocated and simultaneous are brighter than the peaks of those reconnection events (EB or UV burst) that occur independently of each other. In the same light curves, all of the studied UV bursts show a bursty (meaning intermittent) behavior with sometimes up to five intensity peaks during the observation sequence.

Our observations also show the existence of photospheric and chromospheric absorption lines superimposed on the broadened wings of our NUV and FUV IRIS lines. Specifically, we have found Ni II $139.3 \mathrm{~nm}$ superimposed at $-93 \mathrm{~km} \mathrm{~s}^{-1}$ on the wings of the Si IV $139.4 \mathrm{~nm}$ profiles, as well as Mn I superimposed in the blue wing of the Mg II k profiles. Regarding Ni II, it is visible in some of the UV bursts analyzed here and not in others, as the Si IV profiles are not always broad enough to show this absorption. In those bursts with broad enough wings, as in the September 6 case, the absorption is detected throughout the entire lifetime of the UV brightening. Similarly, the Mn I absorption is found in some of our UV brightenings but not in others (see Table 2). In those bursts whose Mg II profiles present the Mn I absorption, it is detected during some time steps within the burst evolution but not in others (see, e.g., Fig. 3). This variability seems to be related to the presence or absence of the cold gas of the surge, which at times partially covers the UV burst area. Similar absorption features on the Mg II line have been reported by Toriumi et al. (2015) as being due to the recurrent ejection of surges from light bridges. Tian et al. (2016) also observe such absorption lines superimposed on the IRIS lines in the data set they analyze. However, these latter authors attribute the presence of these absorption lines to the low formation height of their UV bursts. The argument presented by these latter authors is that one observes such absorption lines when cold gas is stacked above the UV brightening, and since the Mn I line, for example, is formed in the upper photosphere, the cold gas cloud must be lying at that height and the UV burst must be formed below that layer. However, another reason for the presence of absorption lines superimposed on the broadened UV IRIS lines is possible. Both observations and simulations (Tortosa-Andreu \& MorenoInsertis 2009; Ortiz et al. 2014; de la Cruz Rodríguez et al. 2015b; Ortiz et al. 2016; Nóbrega-Siverio et al. 2016; Hansteen et al. 2017, 2019) describe a scenario where cool, dense, magnetized bubbles of gas rise slowly through the photosphere and the chromosphere, expanding both vertically and horizontally and filling the chromosphere and transition region. In the process, the newly uplifted cool, dense gas pumps the necessary opacity to greater heights, which explains absorption in lines such as Mn I and Ni II.

Tian et al. (2016) suggest that the Mg II lines can be used similarly to $\mathrm{H} \alpha$ for the identification of EBs, because in their EBrelated UV bursts the Mg II wings have a significant brightening but no obvious brightening in the line core is observed. However, our EB-only case (described in Sect. 3.4) shows no sign of brightening in the wings of $\mathrm{Mg}$ II, only a very weak increase in the core, and a marginal increase in the triplet only during the peak intensity of the EB, which would rule out the possibility of using $\mathrm{Mg}$ II to trace EBs in the IRIS database. In addition, all our UV bursts leave an imprint in the Mg II profiles, regardless of whether they have an associated EB or not. On the other hand, we notice strong similarities between $\mathrm{Ca}$ II and $\mathrm{H} \alpha$ in $\mathrm{EBs}$, because the wings of $\mathrm{H} \alpha$ and Ca II follow a similar temporal 

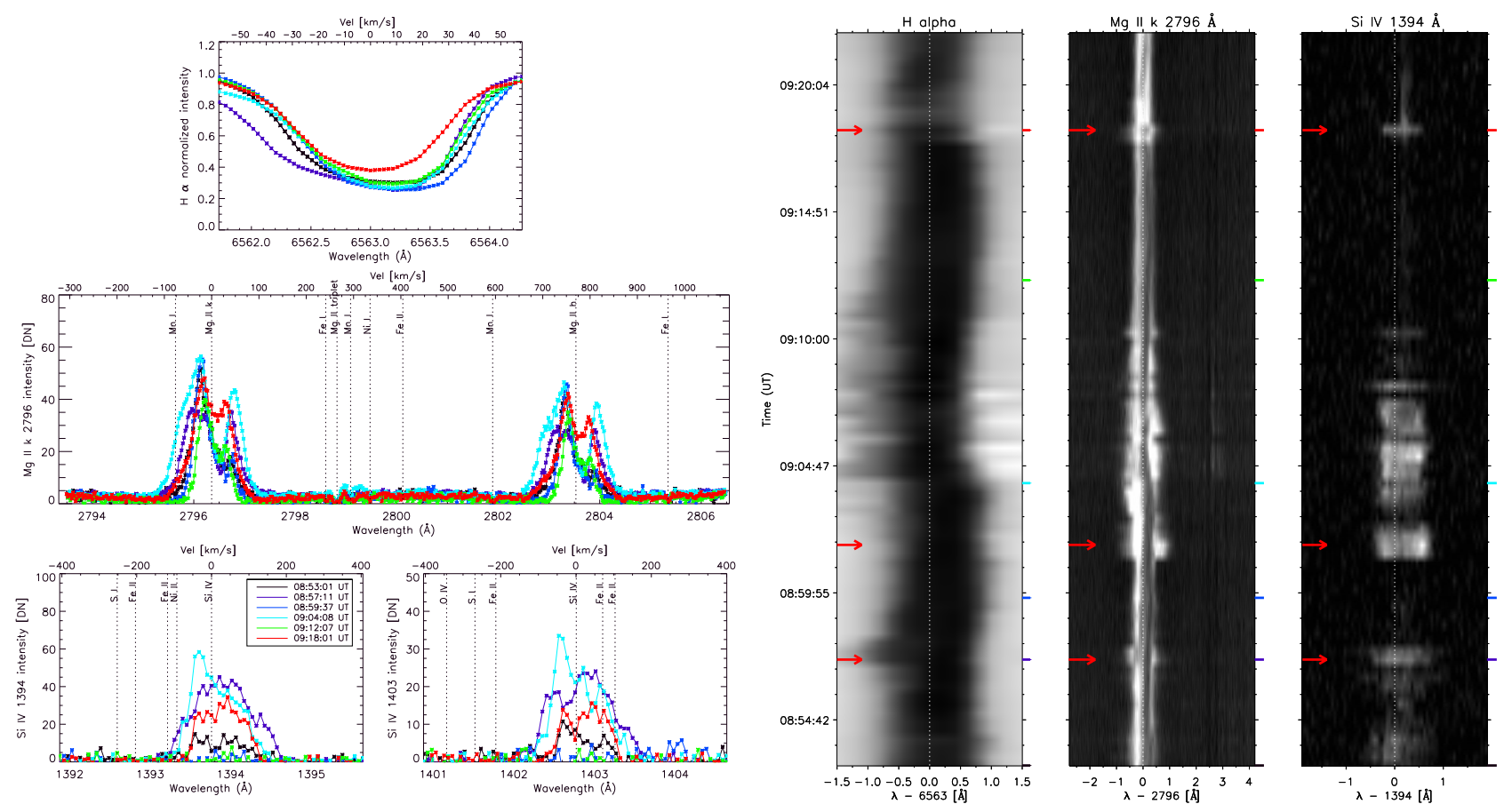

Fig. 15. Same as Fig. 3 but for September 5, 2016, for a case where only a UV burst was present in the FOV. No EB was found that could be related to it. The colored marks in the time-sliced spectra pinpoint to the same times that have been highlighted in the left panel. We have applied a gamma adjustment to the Si IV color table.

evolution and so do the line cores of these spectral lines. However, Vissers et al. (2013) warn that most EBs are also observed in the wings of $\mathrm{Ca}$ II but with a markedly different morphology than in $\mathrm{H} \alpha$.

Table 2 provides evidence that all UV bursts present a surge associated with them. The only case in which we do not observe a surge is the EB-only case on September 5. Since all UV burst cases occur at the site of merging of magnetic fields of opposite polarities, which in turn happen within a region of vigorous flux emergence, we can state that there is a causal relationship between flux emergence and the existence of surges and UV bursts. Nóbrega-Siverio et al. (2017) investigated the details of this relationship and how newly emerged fields interacting with the ambient field produce surges and UV bursts (with and without a coronal response). The numerical simulation of Paper I also produces a surge when a UV burst coexists with an EB. Even though EBs are also caused by flux emergence and merging of opposite polarities, a clear EB-surge connection has not been established (e.g., Rutten et al. 2013; Vissers et al. 2013) and our EB-only case, even though not statistically significant, points in the same direction.

To complete the analysis reported in this work, we studied the morphological and dynamical properties of one of our extended UV brightenings, examining the variations of these properties across the burst area in detail. While a few previous works (Toriumi et al. 2017; Vissers et al. 2019) present results on the positional dependence of the properties of bright points and reconnection events, these works do not carry out an extensive and detailed characterization of the variation of their properties with position. Here we take into account the whole extension of the UV brightening and consider the physical properties at each pixel within the burst domain. Different parts of the UV burst are characterized by different Si IV profile shapes: singlepeaked, double-peaked, or a blend of both, that is, single-peaked with a lesser component in one of the wings. At the same time, when analyzing the velocities obtained from the Si IV $140.3 \mathrm{~nm}$ spectra and their spatial distribution we realized that the burst domain has three different zones: a redshifted area closer to disk center of $1^{\prime \prime}$ width, a blueshifted area in the center of the UV burst domain slightly less than $1^{\prime \prime}$ in width, and an area mainly at rest on the right-hand side of the burst extension. The redshifted zone contains mostly double-peaked profiles while the central blueshifted zone contains mainly either single-peaked profiles or single-peaked profiles with a satellite component in one wing. Finally, the area where the Si IV profiles are at rest is totally populated by single-peaked profiles. This fact could be due to projection effects where bidirectional flows (represented by double-peaked profiles) are more easily observed closer to disk center, or is simply due to the magnetic topology of each particular reconnection event. Maximum Si IV velocities reach blueshifts of $-50 \mathrm{~km} \mathrm{~s}^{-1}$ and redshifts of $100 \mathrm{~km} \mathrm{~s}^{-1}$. The highest velocities are attained before and during the peak of maximum intensity of the UV burst, decreasing afterwards. When comparing the morphology of the burst in various spectral features like the $\mathrm{Mg}$ II $\mathrm{k} 2$ and $\mathrm{k} 3$ peaks or Si IV we also note significant differences in size and shape: the brightening in NUV is wider than in FUV wavelengths ( $2^{\prime \prime}$ width in the Mg II triplet versus $1^{\prime \prime}$ in SiIV). We believe this is due to the decrease of plasma- $\beta$ with height: (overshooting) convective motions play a significant role near the photosphere, but the magnetic field is completely dominant at greater heights where the plasma densities are much lower and temperatures high enough to allow the emission of transition region lines such as Si IV.

The analysis of the morphological, spectral, temporal, and dynamical properties of an extended UV burst yields the picture of a nearly vertical current sheet oriented in the $y$ direction of the FOV. Along this current sheet, nonstationary magnetic reconnection and plasmoids occur. As a result, turbulence and hot irregular bidirectional jets are produced, with downflows on the leftward side of the UV burst and upflows in the central-rightward area 
of the burst. These results agree with, complete, and support the scenario set by the numerical simulations of Paper I.

The works by Peter et al. (2014), Vissers et al. (2015), Grubecka et al. (2016), and Tian et al. $(2016,2018)$ place the formation of UV brightenings very low in the solar atmosphere, even at photospheric levels, even though current EB models argue against this because the typical EB diagnostics cannot be formed if the photosphere is heated to such extreme transition region temperatures. Tian et al. (2016) conclude that some of their UV brightenings are not related to EBs, but other UV bursts would actually be indeed EBs because they also show the typical signatures of an EB. In Paper I and this work we propose an alternative scenario: reconnection due to flux emergence does not occur at discrete heights, but along a long (few thousand $\mathrm{km}$ ) tall wall (in more technical words a current sheet) where heating, plasmoids, turbulence, and bidirectional jets are produced. In this case, coexisting EBs and UV bursts would be part of the same reconnection system, but happening far apart vertically. Hansteen et al. (2017) already proposed that EBs form in the photosphere and UV bursts in the mid-chromosphere around $1300 \mathrm{~km}$ higher up (except that this work did not consider co-spatial and co-temporal events but events that are independent from each other). In this way, the same pixel at or close to disk center can exhibit a typical EB behavior when observing in $\mathrm{H} \alpha$ and a typical UV burst shape when observing in IRIS upper chromosphere and transition region spectra. The heating events happen at different heights and this is evidenced by each of the diagnostics.

The observations presented here do not allow us to constrain the exact formation height of UV bursts relative to EBs; that was one of the main results from the numerical experiments of Paper I. Here we demonstrate the clear temporal and spatial relationship between coexisting EBs and UV brightenings, which behave in unison with a certain sequential character. This work complements and gives observational support to Paper I, but also expands the investigation of the properties of UV bursts. For the first time an extended UV burst has been analyzed in detail and important physical characteristics have been derived. This series of articles characterizes the process of flux emergence, the interaction of the new field with itself and the ambient field, and scrutinizes the transient dynamical phenomena resulting from magnetic reconnection, namely the relationship between EBs and UV bursts.

Acknowledgements. The Swedish $1 \mathrm{~m}$ Solar Telescope is operated on the island of La Palma (Spain) by the Institute for Solar Physics of Stockholm University in the Spanish Observatorio del Roque de los Muchachos of the Instituto de Astrofísica de Canarias. The Institute for Solar Physics is supported by a grant for research infrastructures of national importance from the Swedish Research Council (registration number 2017-00625). This research is supported by the Research Council of Norway, project number 250810, and through its Centers of Excellence scheme, project number 262622. We made much use of NASA's Astrophysics Data System Bibliographic Services.

\section{References}

Archontis, V. 2012, Philos. Trans. R. Soc. London Ser. A, 370, 3088

Archontis, V., Moreno-Insertis, F., Galsgaard, K., Hood, A., \& O'Shea, E. 2004, A\&A, 426, 1047

Carlsson, M., Hansteen, V. H., Gudiksen, B. V., Leenaarts, J., \& De Pontieu, B. 2016, A\&A, 585, A4

Cheung, M. C. M., \& Isobe, H. 2014, Liv. Rev. Sol. Phys., 11, 3

de la Cruz Rodríguez, J., Löfdahl, M. G., Sütterlin, P., Hillberg, T., \& Rouppe van der Voort, L. 2015a, A\&A, 573, A40

de la Cruz Rodríguez, J., Hansteen, V., Bellot-Rubio, L., \& Ortiz, A. 2015b, ApJ, 810,145

de la Cruz Rodríguez, J., Leenaarts, J., \& Asensio Ramos, A. 2016, ApJ, 830, L30
De Pontieu, B., Title, A. M., Lemen, J. R., et al. 2014, Sol. Phys., 289, 2733 Dudík, J., Del Zanna, G., Dzif̌cáková, E., Mason, H. E., \& Golub, L. 2014, ApJ, 780, L12

Ellerman, F. 1917, ApJ, 46, 298

Fletcher, L., Dennis, B. R., Hudson, H. S., et al. 2011, Space Sci. Rev., 159, 19

Galsgaard, K., Archontis, V., Moreno-Insertis, F., \& Hood, A. W. 2007, ApJ, 666,516

Grubecka, M., Schmieder, B., Berlicki, A., et al. 2016, A\&A, 593, A32

Guglielmino, S. L., Zuccarello, F., Young, P. R., Murabito, M., \& Romano, P. 2018, ApJ, 856, 127

Guglielmino, S. L., Young, P. R., \& Zuccarello, F. 2019, ApJ, 871, 82

Hansteen, V. H., Archontis, V., Pereira, T. M. D., et al. 2017, ApJ, 839, 22

Hansteen, V., Ortiz, A., Archontis, V., et al. 2019, A\&A, 626, A33

Hong, J., Carlsson, M., \& Ding, M. D. 2017, ApJ, 845, 144

Isobe, H., Tripathi, D., \& Archontis, V. 2007, ApJ, 657, L53

Judge, P. G. 2015, ApJ, 808, 116

Lemen, J. R., Title, A. M., Akin, D. J., et al. 2012, Sol. Phys., 275, 17

Libbrecht, T., Joshi, J., Rodríguez, J. D. L. C., Leenaarts, J., \& Ramos, A. A. 2017, A\&A, 598, A33

MacTaggart, D., Guglielmino, S. L., Haynes, A. L., Simitev, R., \& Zuccarello, F. 2015, A\&A, 576, A4

Moreno-Insertis, F., \& Galsgaard, K. 2013, ApJ, 771, 20

Nóbrega-Siverio, D., Moreno-Insertis, F., \& Martínez-Sykora, J. 2016, ApJ, 822, 18

Nóbrega-Siverio, D., Martínez-Sykora, J., Moreno-Insertis, F., \& Rouppe van der Voort, L. 2017, ApJ, 850, 153

Nóbrega-Siverio, D., Moreno-Insertis, F., \& Martínez-Sykora, J. 2018, ApJ, 858, 8

Olluri, K., Gudiksen, B. V., \& Hansteen, V. H. 2013, ApJ, 767, 43

Ortiz, A., Bellot Rubio, L. R., Hansteen, V. H., de la Cruz Rodríguez, J., \& Rouppe van der Voort, L. 2014, ApJ, 781, 126

Ortiz, A., Hansteen, V. H., Bellot Rubio, L. R., et al. 2016, ApJ, 825, 93

Pariat, E., Aulanier, G., Schmieder, B., et al. 2004, ApJ, 614, 1099

Pereira, T. M. D., Carlsson, M., De Pontieu, B., \& Hansteen, V. 2015, ApJ, 806, 14

Peter, H., Tian, H., Curdt, W., et al. 2014, Science, 346, 1255726

Raouafi, N. E., Patsourakos, S., Pariat, E., et al. 2016, Space Sci. Rev., 201, 1

Reid, A., Mathioudakis, M., Kowalski, A., Doyle, J. G., \& Allred, J. C. 2017, ApJ, 835, L37

Rouppe van der Voort, L., De Pontieu, B., Scharmer, G. B., et al. 2017, ApJ, 851, L6

Roy, J. R. 1973, Sol. Phys., 32, 139

Rutten, R. J., Vissers, G. J. M., Rouppe van der Voort, L. H. M., Sütterlin, P., \& Vitas, N. 2013, J. Phys. Conf. Ser., 440, 012007

Sainz Dalda, A., de la Cruz Rodríguez, J., De Pontieu, B., \& Gošić, M. 2019, ApJ, 875, L18

Scharmer, G. B. 2006, A\&A, 447, 1111

Scharmer, G. B., Bjelksjo, K., Korhonen, T. K., Lindberg, B., \& Petterson, B. 2003a, in Innovative Telescopes and Instrumentation for Solar Astrophysics, ed. S. L. Keil, S. V. Avakyan, Proc. SPIE, 4853, 341

Scharmer, G. B., Dettori, P. M., Lofdahl, M. G., \& Shand, M. 2003b, in Innovative Telescopes and Instrumentation for Solar Astrophysics, eds. S. L. Keil, \& S. V. Avakyan, Proc. SPIE, 4853, 370

Scherrer, P. H., Schou, J., Bush, R. I., et al. 2012, Sol. Phys., 275, 207

Schmieder, B., Archontis, V., \& Pariat, E. 2014, Space Sci. Rev., 186, 227

Shibata, K. 1997, in Fifth SOHO Workshop: The Corona and Solar Wind Near Minimum Activity, ed. A. Wilson, ESA Spec. Pub., 404, 103

Shibata, K., \& Magara, T. 2011, Liv. Rev. Sol. Phys., 8, 6

Tian, H., Xu, Z., He, J., \& Madsen, C. 2016, ApJ, 824, 96

Tian, H., Zhu, X., Peter, H., et al. 2018, ApJ, 854, 174

Toriumi, S., Katsukawa, Y., \& Cheung, M. C. M. 2015, ApJ, 811, 137

Toriumi, S., Katsukawa, Y., \& Cheung, M. C. M. 2017, ApJ, 836, 63

Tortosa-Andreu, A., \& Moreno-Insertis, F. 2009, A\&A, 507, 949

van Noort, M., Rouppe van der Voort, L., \& Löfdahl, M. G. 2005, Sol. Phys., 228, 191

Vissers, G., \& Rouppe van der Voort, L. 2012, ApJ, 750, 22

Vissers, G. J. M., Rouppe van der Voort, L. H. M., \& Rutten, R. J. 2013, ApJ, 774,32

Vissers, G. J. M., Rouppe van der Voort, L. H. M., Rutten, R. J., Carlsson, M., \& De Pontieu, B. 2015, ApJ, 812, 11

Vissers, G. J. M., de la Cruz Rodríguez, J., Libbrecht, T., et al. 2019, A\&A, 627, A101

Watanabe, H., Vissers, G., Kitai, R., Rouppe van der Voort, L., \& Rutten, R. J. 2011, ApJ, 736, 71

Yang, H., Chae, J., Lim, E.-K., et al. 2013, Sol. Phys., 288, 39

Yokoyama, T., \& Shibata, K. 1995, Nature, 375, 42

Young, P. R., Tian, H., Peter, H., et al. 2018, Space Sci. Rev., 214, 120 